Check for updates

Cite this: RSC Adv., 2019, 9, 40152

Received 2nd October 2019

Accepted 20th November 2019

DOI: $10.1039 / \mathrm{c} 9 \mathrm{ra} 08002 \mathrm{c}$

rsc.li/rsc-advances

\section{Pd-catalyzed intramolecular addition of active methylene compounds to alkynes with subsequent cross-coupling with (hetero)aryl halides $\uparrow$}

\author{
Aleksandra Błocka, ${ }^{a}$ Paweł Woźnicki, (DD b Marek Stankevič (iD b \\ and Wojciech Chaładaj iD *a
}

\begin{abstract}
We report an efficient protocol for tandem Pd-catalyzed intramolecular addition of active methylene compounds to alkynes, followed by subsequent cross-coupling with (hetero)aryl bromides and chlorides. The reaction proceeds under mild conditions, providing excellent functional group tolerance, including unprotected $\mathrm{OH}, \mathrm{NH}_{2}$ groups, enolizable ketones, or a variety of heterocycles. Mechanistic studies point towards a catalytic cycle involving oxidative addition, intramolecular nucleophilic addition to the $\mathrm{Pd}(\Perp)$ activated alkyne, and reductive elimination, with 5-exo-dig cyclization being the rate limiting step.
\end{abstract}

\section{Introduction}

Palladium complexes emerge as some of the most versatile homogenous catalysts with a myriad of applications in both academic and industrial research. The most prominent area of palladium catalysis, awarded with the 2010 Nobel Price to R. Heck, A. Suzuki, and E. Negishi, ${ }^{1}$ covers cross-couplings of (hetero)aryl or vinyl(pseudo)halides with nucleophilic or organometallic partners. High efficiency of these and many other processes (e.g. Wacker oxidation) arises from the facile interconversion of palladium oxidation states through twoelectron redox chemistry. Besides the most widespread $\operatorname{Pd}(0) /$ $\mathrm{Pd}(\mathrm{II})$ cycle, palladium is also able to enter radical processes or to serve as a carbophilic Lewis acid in redox-neutral transformations. The ability to mediate mechanistically distinct transformations makes palladium the catalyst of choice for the design of tandem reactions in which a single metal complex catalyzes a sequence of transformations. ${ }^{2}$ In our research, we are focused on the development of tandem processes combining the nucleophilic addition to alkynes and subsequent cross-coupling, which give the access to a wide set of carbo- and heterocyclic systems. ${ }^{3}$ In contrast to cross-coupling reactions, these transformations are highly underdeveloped and suffer from harsh reaction conditions (e.g. the use of strong bases), narrow substrate scope (usually limited to active aryl iodides), and poor functional group tolerance, as well as insufficient mechanistic understanding.

${ }^{a}$ Institute of Organic Chemistry, Polish Academy of Sciences, Kasprzaka 44/52, 01-224 Warsaw, Poland. E-mail: wojciech.chaladaj@icho.edu.pl

${ }^{b}$ Department of Organic Chemistry, Faculty of Chemistry, Marie Curie-Skłodowska University in Lublin, Gliniana 33, 20-614 Lublin, Poland

$\dagger$ Electronic supplementary information (ESI) available. See DOI: 10.1039/c9ra08002c
In the late 1980s, Gore disclosed seminal works on a novel Pd-catalyzed dicarbofunctionalization of unsaturated $\mathrm{C}-\mathrm{C}$ systems through arylation with iodobenzene and intramolecular nucleophilic additions of malonates to alkylidenecyclopropanes or alkenes. ${ }^{4}$ In subsequent accounts, the authors reported a sequential 5-exo-dig cyclization of malonates and $\beta$ ketoesters tethered to the alkyne moiety, followed by coupling with aryl iodides. ${ }^{5}$ The scope of the methodology was further extended to the use of haloalkynes, ${ }^{6}$ allyl halides and acetates ${ }^{7}$ as coupling partners. Recently, we have developed a protocol enabling the effective reaction of much less active aryl bromides with acetylenic $\beta$-ketoesters. ${ }^{8}$ A similar strategy, utilizing a 5endo-dig cyclization has also been applied to the synthesis of cyclopentenes $^{9}$ and indenes. ${ }^{10}$ Propargylmalonates led to substituted cyclopropanes via analogous cyclization/coupling protocol. ${ }^{11}$ On the other hand, propargyl- $\beta$-ketoesters underwent 5-exo-dig oxocyclization/coupling, leading to the formation of substituted furan systems due to ambident nature of enolates of $\beta$-ketoesters. ${ }^{\mathbf{1 2}}$ Interestingly, the analogous transformation involving homopropargyl- $\beta$-ketoesters possessing an internal or terminal alkyne motif clearly led to either cyclopentenes ${ }^{9}$ or dihydropyranes, ${ }^{13}$ respectively.

The vast majority of the known methodologies utilizing sequential Pd-catalyzed nucleophilic cyclization and cross coupling are limited to aryl iodides. Moreover, the functional group compatibility appeared very narrow, which could possibly arise from the use of a strong base. Recently, we have addressed these challenges in a transformation involving acetylenic $\beta$ ketoesters which readily undergo cyclization. Extension of the scope with respect to activated methylene compounds still awaits investigation. Although there are examples of such transformations involving derivatives of ketoesters and malonates (with active aryl iodides), to the best of our knowledge, cyclization/coupling of haloarenes with acetylenic derivatives of 
Table 1 Optimization of the reaction conditions for benchmark reaction

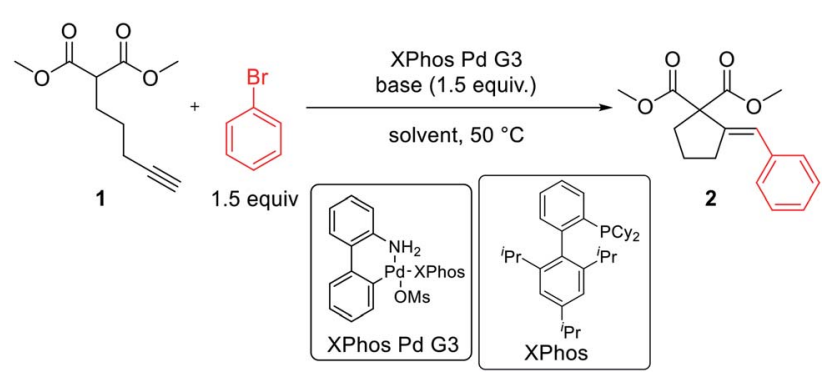

\begin{tabular}{llllll}
\hline Entry & Solvent & Base & Time & Cat. loading & Yield $^{a}$ \\
\hline 1 & Toluene & $\mathrm{K}_{3} \mathrm{PO}_{4}$ & $4 \mathrm{~h}$ & $1 \mathrm{~mol} \%$ & $1 \%$ \\
2 & Dioxane & $\mathrm{K}_{3} \mathrm{PO}_{4}$ & $4 \mathrm{~h}$ & $1 \mathrm{~mol} \%$ & $3 \%$ \\
3 & THF & $\mathrm{K}_{3} \mathrm{PO}_{4}$ & $4 \mathrm{~h}$ & $1 \mathrm{~mol} \%$ & $2 \%$ \\
4 & $\mathrm{MeCN}$ & $\mathrm{K}_{3} \mathrm{PO}_{4}$ & $4 \mathrm{~h}$ & $1 \mathrm{~mol} \%$ & $8 \%$ \\
5 & DMSO & $\mathrm{K}_{3} \mathrm{PO}_{4}$ & $4 \mathrm{~h}$ & $1 \mathrm{~mol} \%$ & $47 \%$ \\
6 & $\mathrm{DMF}$ & $t-\mathrm{BuOK}$ & $4 \mathrm{~h}$ & $1 \mathrm{~mol} \%$ & $0 \%$ \\
7 & $\mathrm{DMF}$ & $\mathrm{KHMDS}$ & $4 \mathrm{~h}$ & $1 \mathrm{~mol} \%$ & $0 \%$ \\
8 & $\mathrm{DMF}$ & $\mathrm{K}_{2} \mathrm{CO}_{3}$ & $4 \mathrm{~h}$ & $1 \mathrm{~mol} \%$ & $22 \%$ \\
9 & $\mathrm{DMF}$ & $\mathrm{K}_{3} \mathrm{PO}_{4}$ & $4 \mathrm{~h}$ & $1 \mathrm{~mol} \%$ & $22 \%$ \\
10 & $\mathrm{DMF}$ & $\mathrm{K}_{3} \mathrm{PO}_{4}$ & $4 \mathrm{~h}$ & $1 \mathrm{~mol} \%$ & $61 \%$ \\
11 & $\mathrm{DMF}$ & $\mathrm{K}_{3} \mathrm{PO}_{4}$ & $24 \mathrm{~h}$ & $2 \mathrm{~mol} \%$ & $90 \%$
\end{tabular}

${ }^{a}$ Determined by GC with mesitilene as an internal standard.

malononitrile, cyanoacetates, diketones, as well as substrates bearing organophosphorus electron-withdrawing functions have not been reported.
Here, we report an efficient protocol for tandem Pd-catalyzed intramolecular addition of active methylene compounds to alkynes and subsequent cross-coupling with (hetero)aryl bromides and chlorides. The methodology features excellent tolerance for functionalities present in either reaction partner.

\section{Results and discussion}

The reaction of dimethyl pent-4-yn-1-ylmalonate 1 with bromobenzene was chosen as a model transformation for the development of the reaction conditions. First, a range of Pdcomplexes of mono- and diphosphine ligands were examined using 3rd-generation Buchwald-type palladacyclic system as a platform in order to identify an active catalyst system. Optimization revealed XPhos Pd G3 as the pre-catalyst of choice. Then, the benchmark reaction was evaluated against various reaction conditions, including base, solvent, catalyst loading, temperature, and time, among others (Table 1$).{ }^{14}$ A polar aprotic solvent appeared to be crucial for the efficiency of the cyclization. Reactions carried out in moderately polar, or nonpolar solvents (e.g. dioxane, THF, toluene) failed to proceed at all, or competitive Sonogashira coupling was observed. The best results were achieved for the reaction run for $24 \mathrm{~h}$ at $50{ }^{\circ} \mathrm{C}$ in DMF with potassium phosphate as the base. $2 \mathrm{~mol} \%$ of palladium complex was necessary to achieve a high yield of desired product 2 .

With satisfactory conditions developed for the model substrate, we proceeded to investigate the scope of the reaction. First, we examined the performance of various aryl and heteroaryl bromides in the reaction with malonate $\mathbf{1}$ (Table 2 ).

Table 2 Substrate scope: aryl bromides ${ }^{a}$
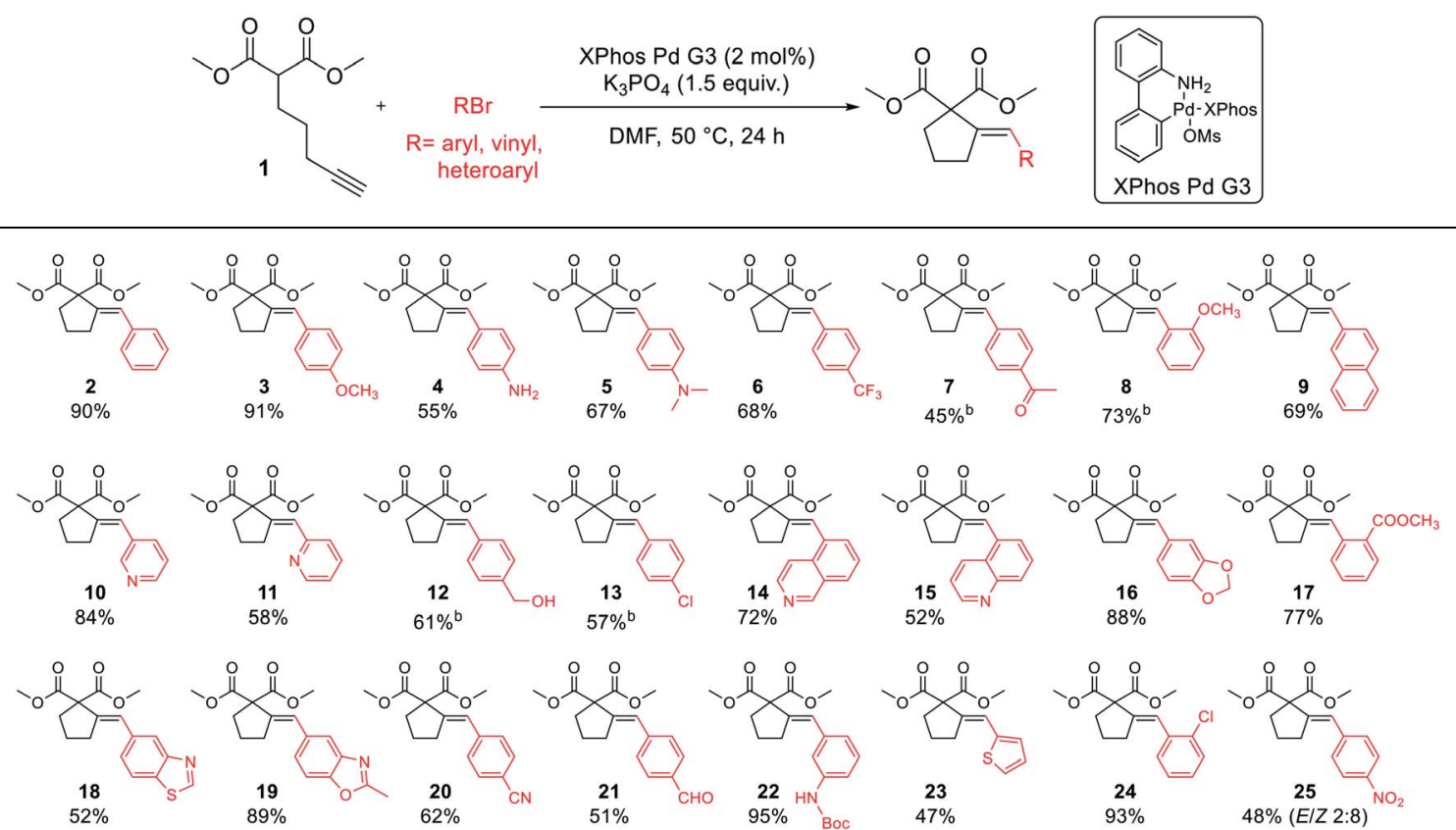

\footnotetext{
${ }^{a}$ Reaction conditions: dimethyl pent-4-yn-1-ylmalonate $\mathbf{1}(0.400 \mathrm{mmol})$, aryl bromide (0.500 mmol), $\mathrm{K}_{3} \mathrm{PO}_{4}(0.600 \mathrm{mmol})$, XPhos Pd G3 (8.0 $\mu$ mol, $2 \mathrm{~mol} \%)$, DMF $(1 \mathrm{ml}), 50^{\circ} \mathrm{C}, 24 \mathrm{~h} .{ }^{b}$ Run for $4 \mathrm{~h}$.
} 
Both electron-rich and electron-poor bromoarenes smoothly underwent the reaction, affording the expected products with good to excellent yields and complete stereoselectivity on the olefinic bond. A range of functional groups including, inter alia, unprotected amines (4), alcohols (12), aldehydes (21), nitriles (20), nitro (25), carbamates (22), or enolizable ketones (7) were well tolerated. Furthermore, sterically hindered $o$-substituted bromo(hetero)arenes also proved to be complementary reaction partners $(\mathbf{8}, \mathbf{1 7}, \mathbf{2 4})$. The use of various heteroaryl bromides enabled the introduction of the heterocyclic moiety to the product (10-11, 14-16, 18-19, 23), including pharmaceutically relevant $\mathrm{N}$-heterocyclic motifs (10-11, 14-16, 18-19).

Next, we proceeded to examine the scope and limitations with respect to various acetylenic active methylene compounds (Table 3). Selected derivatives of malonates, cyanoacetates, cyanomalonates, $\beta$-ketoesters, and 1,3-diketones were subjected to the reaction with both electron-poor and electron-rich bromoarenes - bromobenzene, $p$-bromoanisole, and $p$-bromobenzonitrile. Transformations with more sterically hindered $i$ propyl and $t$-butyl malonates delivered the expected products (26-31), although with diminished yields, compared to the less sterically demanding methyl malonate $\mathbf{1}$.

Table 3 Substrate scope: acetylenic active methylene compounds ${ }^{a}$

(1)
$30 \mathrm{R}=\mathrm{OMe} 23 \%$ c $33 \mathrm{R}=\mathrm{OMe} 96 \%$

The considerably more $\mathrm{C}-\mathrm{H}$ acidic cyanoacetates, cyanomalonates, and $\beta$-ketoesters appeared to be the more reactive substrates, usually providing the appropriate products (32-43) with very good yields (70-96\%). The only exception was a reaction of electron-deficient bromoarenes with cyanomalonate and $t$-butyl cyanoacetate, which afforded products (34 and 40) with moderate yields (33-40\%). Notably, reactions involving electron-deficient bromoarenes and all of the above-mentioned acetylenic substrates proceeded with high, but not complete diastereoselectivity $(E / Z$ selectivity). All reactions involving electronically neutral, or electron-rich bromoarenes provided complete selectivity.

Next, we investigated various phosphorus-substituted acetylenes as potential reaction partners. We were pleased to find that esters, ketones, and nitriles bearing phosphoryl or phosphinoyl functions entered the reaction with bromobenzene, affording the target cyclopentanes (49-54) with moderate to good yields and complete diastereoselectivity. Compound 49 was isolated with a low yield due to difficulties in the isolation and purification.

Finally, we were pleased to find that the developed protocol is also applicable to the remarkably less active aryl chlorides (Table 4). Both electron-rich and electron-deficient chloroarenes, as well as heteroaryl chlorides (2-chloropyridine) entered the reaction, yielding the expected products in moderate to good yields (39-69\%). Interestingly, electron-deficient chloroarenes gave products with low diastereoselectivity, in contrast to their corresponding aryl bromides which provided the products as single isomers (except 4-nitrobromobenzene).

The postulated mechanism, based on the observations of the reaction outcome, several control experiments, and literature data, is depicted in Scheme 1. First, the bromoarene undergoes fast oxidative addition to $\operatorname{Pd}(0)$ complex 57 (formed upon the activation of the precatalyst with a base) ${ }^{15}$ leading to the

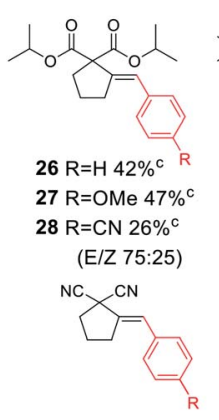

$38 \mathrm{R}=\mathrm{H} 87 \%^{\mathrm{b}}$ $39 \mathrm{R}=\mathrm{OMe} 91 \% \mathrm{~b}$

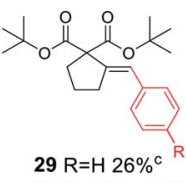

$31 \mathrm{R}=\mathrm{CN} 35 \%$

$$
\text { (E/Z 35:65) }
$$

$34 \mathrm{R}=\mathrm{CN} 33 \%$ (E/Z 90:10)
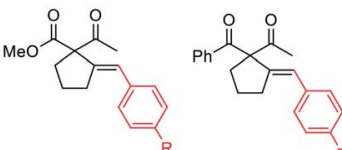

$40 \mathrm{R}=\mathrm{H} 80 \% \mathrm{~d}^{\mathrm{R}} \quad 43 \mathrm{R}=\mathrm{H} 45 \% \mathrm{c}^{\mathrm{R}}$

$42 \mathrm{R}=\mathrm{CN} 75 \%^{\mathrm{d}} \quad 45 \mathrm{R}=\mathrm{CN} 40 \%^{\mathrm{c}}$ (E/Z 91:9) $\quad(E / Z$ 90:10)

$48 \mathrm{R}=\mathrm{CN} 40 \%^{\mathrm{c}}$ (E/Z 90:10)

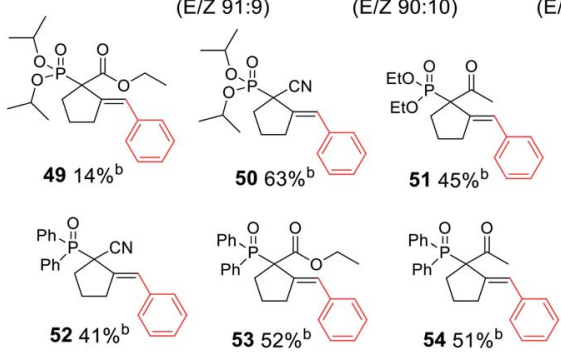

${ }^{a}$ Reaction conditions: acetylenic active methylene compound $(0.400$ $\mathrm{mmol})$, aryl bromide $(0.500 \mathrm{mmol}), \mathrm{K}_{3} \mathrm{PO}_{4}(0.600 \mathrm{mmol})$, XPhos Pd G3 (8.0 $\mathrm{mmol}, 2 \mathrm{~mol} \%)$, DMF $(1 \mathrm{ml}), 50{ }^{\circ} \mathrm{C}, 24 \mathrm{~h} .{ }^{b}$ Run for $4 \mathrm{~h} .{ }^{c}$ Run at $80{ }^{\circ} \mathrm{C}$ for $24 \mathrm{~h} .{ }^{d}$ Run at $50{ }^{\circ} \mathrm{C}$ for $2 \mathrm{~h}$.

\footnotetext{
${ }^{a}$ Reaction conditions: dimethyl pent-4-yn-1-ylmalonate $1(0.400 \mathrm{mmol})$, aryl chloride $(0.500 \mathrm{mmol}), \mathrm{K}_{3} \mathrm{PO}_{4}(0.600 \mathrm{mmol})$, XPhos Pd G3 $(8.0 \mu \mathrm{mol}$, $2 \mathrm{~mol} \%)$, DMF (1 ml), $80{ }^{\circ} \mathrm{C}, 24 \mathrm{~h}$.
} 


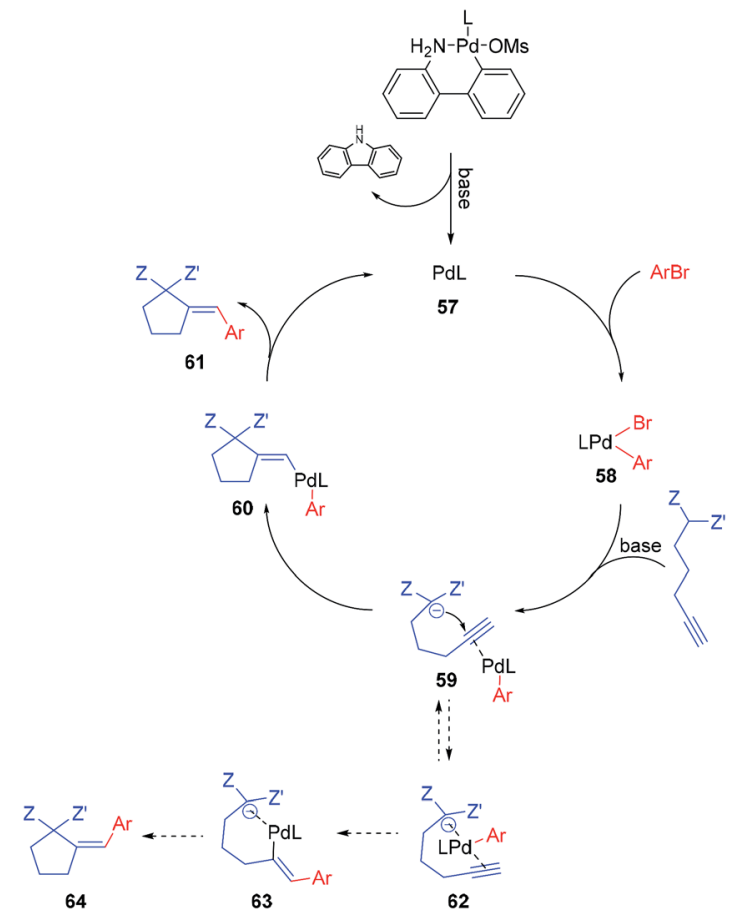

Scheme 1 Plausible mechanism.

formation of aryl-Pd(II) species $\mathbf{5 8}$ which coordinates to the alkyne moiety. Then, intramolecular nucleophilic addition to the activated unsaturated system occurs, providing vinyl-Pd(II) species 60 which undergoes facile reductive elimination affording the expected product $\mathbf{6 1}$ and reconstituting the $\operatorname{Pd}(0)$ complex 57. Although the above mechanism seems viable for the majority of the investigated reactions, for some specific combinations of substrates, alternative scenarios should also be considered. For instance, the formation of chelate $\mathbf{6 2}$ (possibly being in equilibrium with 59), in which palladium is bound by both alkyne and active methylene moieties, could facilitate the insertion of the Pd-arene to the alkyne (syn-carbometallation), and thus rationalize the formation of some amount of another diastereoisomer of the product with altered configuration at the exocyclic double bond (64).

Oxidative addition to $\mathrm{Pd}(0)$ ligated to a single electron-rich monophosphine is fast. In fact, oxidative addition of bromoarene to XPhos- $\mathrm{Pd}(0)$ complex proceeds within minutes at room temperature, as observed by ${ }^{31} \mathrm{P}$ NMR spectroscopy. Reductive elimination from Pd complexes of sterically demanding ligands is also facile. In particular, we have recently shown that the reductive elimination is not a rate-limiting step in the XPhos-Pd-catalyzed tandem cyclization/coupling of $\varepsilon-$ acetylenic $\beta$-ketoesters with aryl bromides (Scheme 2a). ${ }^{8}$ The tandem reaction of ketoester $\mathbf{6 5}$ with bromobenzene is much slower than Negishi coupling of compound 66 with diphenylzinc, both proceeding through reductive elimination from a common intermediate 67 . This points towards the conclusion that the cyclization step is a bottleneck of the transformation. In order to shed more light on the influence of the structure of reagents on the reaction outcome, we compared the rate of reactions of bromobenzene with three acetylenic substrates derivatives of malonate $1, \beta$-ketoester 65 , and $\beta$-diketone 68 (Scheme $2 \mathrm{~b}$ ). As expected, malonate 1 reacted significantly slower than ketoester $\mathbf{6 5}$, providing the corresponding product in only $21 \%$ yield after $1 \mathrm{~h}$, compared to $90 \%$ for 65 . This is due to considerably lower $\mathrm{C}-\mathrm{H}$ acidity of the malonate. Surprisingly, under identical conditions, the more $\mathrm{C}-\mathrm{H}$ acidic $\beta$-diketone $\mathbf{6 8}$ delivered the product with only $14 \%$ yield. Competition experiments, involving pairs of acetylenic substrates ( 1 equiv. of each) and bromobenzene ( 1 equiv.) were also conducted (Scheme 2c). A reaction involving ketoester $\mathbf{6 5}$ and malonate $\mathbf{1}$ delivered only the product of the cyclization/coupling of $\mathbf{6 5}$, demonstrating the huge difference in their reactivity. Despite diketone 68 reacting slower than malonate in a parallel experiment (see: Scheme 2b), in the competition experiment it provided higher yield of the corresponding product ( $60 \%$ and $31 \%$, respectively). Similarly, the cyclization of ketoester and diketone occurred at comparable rates under the competition conditions $(42 \%$ and $27 \%$, respectively), in contrast to the parallel experiment ( $90 \% v s$. $14 \%)$. The remarkably slow reaction of diketone 68 could be attributed either to the lower nucleophilicity of its enolate due to extended resonance stabilization, or the capability for the formation of stable complexes with palladium. ${ }^{16}$ The relatively stable palladium complex with diketone (or its anion) could possibly be in tautomeric equilibrium with Pd-alkyne complex suitable for intramolecular nucleophilic addition leading to 61 . Thus, the involvement of arylpalladium 58 in complexation with diketone 68 could make it less available for the catalytic transformation of the more reactive ketoester $\mathbf{6 5}$ in the competition experiment.

Competition experiments of malonate 1 with pairs of electronically divergent bromoarenes revealed the preference for the reaction with the more electron-deficient substrate (Scheme $2 \mathrm{e})$. This stays in contrast with the outcome of the parallel experiments of 1 with each of the above bromoarenes showing comparable rates (Scheme 2d). Apparently, oxidative addition is not a rate limiting step, although in control experiments it determines the ratio of aryl-Pd(II) intermediates, which in turn dictates the final product distribution.

Another factor used for better understanding the reaction mechanism is the stereochemical outcome of the transformation. All of the reactions with malonates proceeded with complete diastereoselectivity, arising from anti-carbopalladation of the alkyne moiety. Similarly, other acetylenic active methylene compounds delivered the corresponding products as single isomers, unless electron-deficient bromoarenes (e.g. pbromobenzonitrile) were used as coupling partners. In this case, the isomer with the alternate configuration on the double bond was formed to some extent, suggesting an alternative pathway for these sets of substrates (Scheme 1, dashed lines).

\section{Experimental}

All manipulations were performed in a nitrogen-filled glovebox or under an argon atmosphere using Schlenk techniques, unless mentioned otherwise. Flash chromatography was performed using Merck silica gel 60 (230-400 mesh). TLC analysis 
a) Control experiment - fast reductive elimination
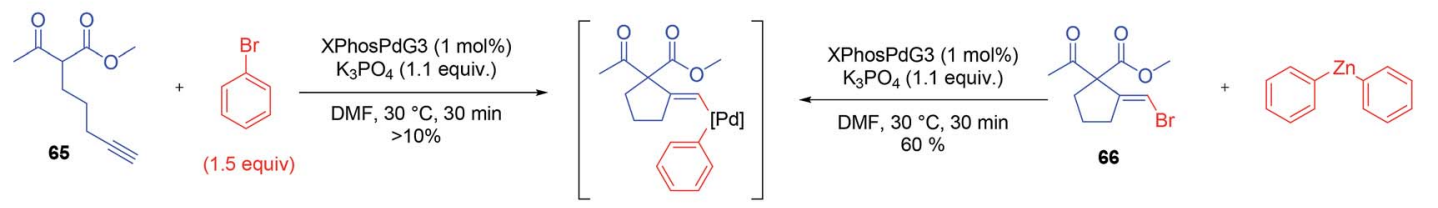

common intermediate

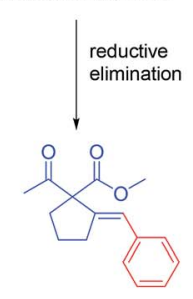

b) Control experiment - comparison of reactivity of acetylenic active methylenes
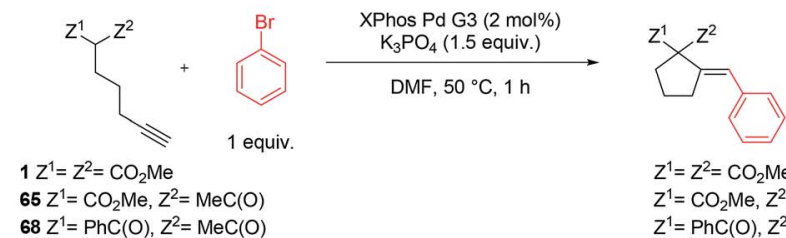

$Z^{1}=Z^{2}=\mathrm{CO}_{2} \mathrm{Me} \quad 21 \%$

$Z^{1}=\mathrm{CO}_{2} \mathrm{Me}, Z^{2}=\mathrm{MeC}(\mathrm{O}) \quad 90 \%$

$Z^{1}=\operatorname{PhC}(O), Z^{2}=\operatorname{MeC}(O) \quad 14 \%$

c) Competition experiment - comparison of reactivity of acetylenic active methylenes
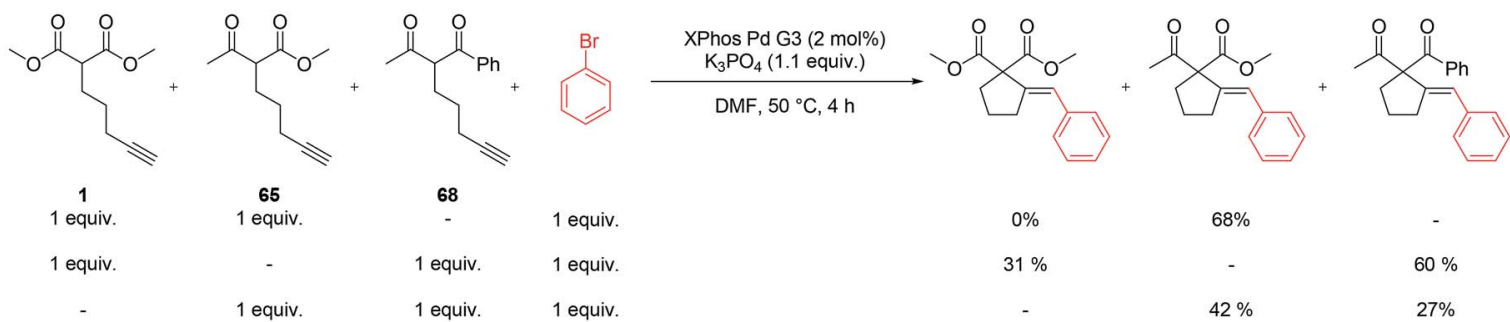

$0 \%$

$68 \%$

$31 \%$

$-$

$60 \%$

1 equiv.

1 equiv.

$-$

$42 \%$

$27 \%$

d) Control experiment - comparison of reactivity of bromoarenes
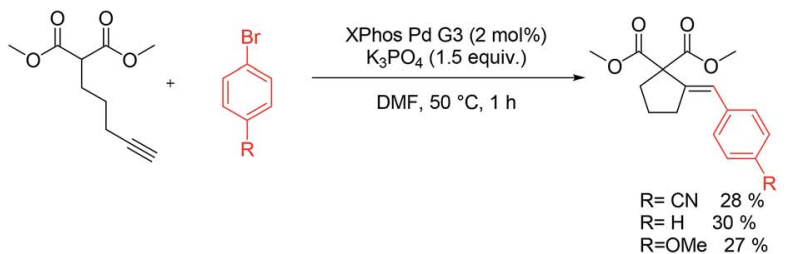

e) Control experiment - comparison of reactivity of bromoarenes
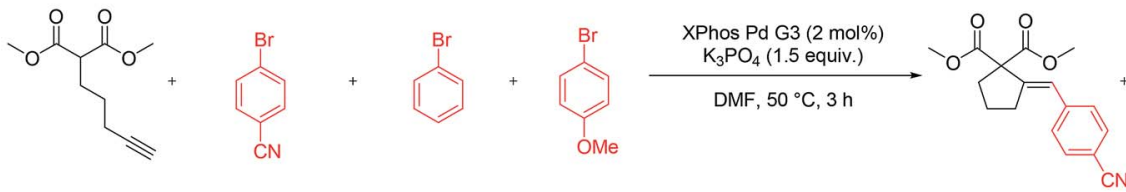

$\begin{array}{lc}1 \text { equiv. } & - \\ 1 \text { equiv. } & 1 \text { equiv. } \\ 1 \text { equiv. } & 1 \text { equiv. }\end{array}$
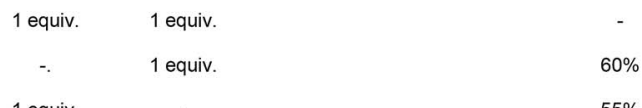

$42 \%$
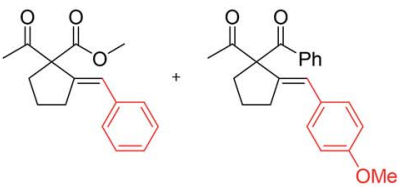

$60 \%$

$-$

$32 \%$

$5 \%$

$55 \%$

$7 \%$

Scheme 2 Control experiments.

of reaction mixtures was performed on Merck silica gel 60 F254 TLC plates and visualized with cerium molybdate stain (Hanessian's stain). ${ }^{1} \mathrm{H},{ }^{13} \mathrm{C}\{1 \mathrm{H}\}$, and ${ }^{19} \mathrm{~F}$ NMR spectra were recorded with a Bruker AV 400 spectrometer. ${ }^{1} \mathrm{H}$ and ${ }^{13} \mathrm{C}$ chemical shifts are given in ppm relative to TMS. Solvent signals were used as references $\left(\mathrm{CDCl}_{3} \delta_{\mathrm{H}}=7.26 \mathrm{ppm}, \delta_{\mathrm{C}}=\right.$ 
$77.0 \mathrm{ppm}$ ) and the chemical shift converted to the TMS scale. Coupling constants $(J)$ are reported in $\mathrm{Hz}$, and the following abbreviations were used to denote multiplets: $\mathrm{s}=$ singlet, $\mathrm{d}=$ doublet, $\mathrm{t}=$ triplet, $\mathrm{q}=$ quartet, quint $=$ quintet, $\mathrm{m}=$ multiplet (denotes a complex pattern), $\mathrm{dd}=$ doublet of doublets, $\mathrm{dt}$ $=$ doublet of triplets and $\mathrm{br}=$ broad signal. Infrared spectra were recorded with a Jasco FTIR-6200 spectrometer. Electron ionization high-resolution mass spectra (EI-HR) were recorded with an Autospec Premier (Waters Inc) mass spectrometer using the narrow-range high-voltage scan technique with lowboiling perfluorokerosene (PFK) as internal standard. Samples were introduced by using a heated direct insertion probe. Electrospray ionization high-resolution mass spectra (ESI-HR) were recorded with MALDISynapt G2-S HDMS (Waters Inc) mass spectrometer equipped with an electrospray ion source and q-TOF type mass analyzer. ESI-MS spectra were recorded in the positive ion mode (source parameters: capillary voltage $3.15 \mathrm{kV}$, sampling cone $25 \mathrm{~V}$, source temperature $120^{\circ} \mathrm{C}$, desolvation temperature $150{ }^{\circ} \mathrm{C}$ ).

Unless otherwise noted, all commercially available compounds (ABCR, Acros, Fluorochem, TCI, Sigma-Aldrich, Strem) were used as received. Phosphine ligands were purchased from Aldrich or Fluorochem, $\mathrm{Pd}(\mathrm{OAc})_{2}$ was purchased from Strem. Buchwald-type 3rd-generation palladacyclic precatalysts (Ligand Pd G3) were prepared following literature procedures, ${ }^{\mathbf{1 5}}$ and showed similar reactivity to the commercial samples (XPhos Pd G3 was compared with commercial samples). Dimethyl pent-4-yn-1-ylmalonate 1 and other acetylenic active methylene compounds were synthesized by alkylation of dimethyl malonate or other $\mathrm{C}-\mathrm{H}$ acids with 1iodo-pentyne, according to typical literature procedures.

General procedure A for Pd-catalyzed carbocyclizationcoupling of aryl bromides with acetylenic active methylene compounds

In a drybox, a $4 \mathrm{ml}$ screw-cap vial was charged with XPhos Pd G3 (6.8 mg, $8 \mu \mathrm{mol}$ ), aryl halide (0.5 mmol), $\mathrm{K}_{3} \mathrm{PO}_{4}(127.2 \mathrm{mg}, 0.6$ $\mathrm{mmol}), \mathrm{DMF}(1 \mathrm{ml})$, and a magnetic stirring bar. Then, acetylenic active methylene compound (e.g. dimethyl pent-4-yn-1ylmalonate 1) was added $(0.4 \mathrm{mmol})$, the vial was tightly sealed and removed from drybox. The reaction mixture was stirred for $24 \mathrm{~h}$ at $50{ }^{\circ} \mathrm{C}$ in a heating block, then cooled to room temperature, quenched with $20 \mathrm{ml}$ of an $\mathrm{NH}_{4} \mathrm{Cl}$ solution, added to $10 \mathrm{ml}$ of water, and extracted with MTBE $(3 \times 10 \mathrm{ml})$. The combined organic phases were dried with $\mathrm{Na}_{2} \mathrm{SO}_{4}$, filtered, and concentrated. The crude product was purified by column chromatography on silica gel.

\section{Dimethyl (2E)-2-benzylidenecyclopentane-1,1-dicarboxylate (2)}

Prepared in reaction of dimethyl 4-pentenylmalonate and bromobenzene following general procedure (105 $\mathrm{mg}, 90 \%)$ or in reaction with chlorobenzene following modified general procedure (run at $\left.80{ }^{\circ} \mathrm{C}\right)(71 \mathrm{mg}$, yield $65 \%)$. Product was isolated as oil after column chromatography on silica gel ( $15 \mathrm{~g}$, hex/ AcOEt 95 : 5). ${ }^{1} \mathrm{H}$ NMR (400 MHz, $\left.\mathrm{CDCl}_{3}\right) \delta 7.37-7.30(\mathrm{~m}, 4 \mathrm{H})$, $7.24-7.19(\mathrm{~m}, 1 \mathrm{H}), 6.71(\mathrm{t}, J=2.4 \mathrm{~Hz}, 1 \mathrm{H}), 3.78(\mathrm{~s}, 6 \mathrm{H}), 2.72(\mathrm{td}, J$
$=7.2,2.5 \mathrm{~Hz}, 2 \mathrm{H}), 2.40(\mathrm{t}, J=6.9 \mathrm{~Hz}, 2 \mathrm{H}), 1.84(\mathrm{p}, J=7.1 \mathrm{~Hz}$, $2 \mathrm{H}) ;{ }^{13} \mathrm{C}$ NMR (101 MHz, $\mathrm{CDCl}_{3}$ ) $\delta$ 171.4, 141.0, 137.6, 128.7, 128.2, 127.4, 126.8, 65.4, 52.8, 35.7, 32.0, 24.8; IR $\left(\mathrm{CH}_{2} \mathrm{Cl}_{2}\right)$ : 3053, 3024, 2953, 2878, 2842, 1733, 1431, 1263, 1152, 773, $696 \mathrm{~cm}^{-1}$; HRMS (ESI) $m / z$ calcd for $\mathrm{C}_{16} \mathrm{H}_{18} \mathrm{O}_{4} \mathrm{Na} 297.1103$; found 297.1097.

\section{Dimethyl (2E)-2-(4-methoxybenzylidene)cyclopentane-1,1- dicarboxylate (3)}

Prepared in reaction of dimethyl 4-pentenylmalonate and 4bromoanisole following general procedure (110 mg, yield 91\%) or in reaction with 4-chloroanisole following modified general procedure (run at $80{ }^{\circ} \mathrm{C}$ ) (81 $\mathrm{mg}$, yield $67 \%$ ). Product was isolated as oil after column chromatography on silica gel (15 g, hex/ AcOEt 80 : 20). ${ }^{1} \mathrm{H}$ NMR (400 MHz, $\left.\mathrm{CDCl}_{3}\right) \delta 7.31-7.26(\mathrm{~m}, 2 \mathrm{H})$, 6.89-6.84 (m, 2H), $6.63(\mathrm{t}, J=2.6 \mathrm{~Hz}, 1 \mathrm{H}), 3.80(\mathrm{~s}, 3 \mathrm{H}), 3.76(\mathrm{~s}$, $6 \mathrm{H}), 2.69(\mathrm{td}, J=7.2,2.6 \mathrm{~Hz}, 2 \mathrm{H}), 2.38(\mathrm{t}, J=6.9 \mathrm{~Hz}, 2 \mathrm{H}), 1.83(\mathrm{p}$, $J=7.0 \mathrm{~Hz}, 2 \mathrm{H}) ;{ }^{13} \mathrm{C} \mathrm{NMR}\left(101 \mathrm{MHz}, \mathrm{CDCl}_{3}\right) \delta 171.5,158.4,138.7$, 130.4, 129.9, 126.8, 113.6, 65.3, 55.2, 52.7, 35.7, 31.9, 24.8; IR $\left(\mathrm{CH}_{2} \mathrm{Cl}_{2}\right): 2954,2838,1732,1606,1512,1435,1251,1177,1033$, $826 \mathrm{~cm}^{-1}$; HRMS (ESI): $\mathrm{m} / z$ calcd for $\mathrm{C}_{17} \mathrm{H}_{20} \mathrm{O}_{5} \mathrm{Na} 327.1208$; found 327.1196 .

\section{Dimethyl (2E)-2-(4-aminobenzylidene)cyclopentane-1,1- dicarboxylate (4)}

Prepared in reaction of dimethyl 4-pentenylmalonate and 4bromoaniline following general procedure ( $63 \mathrm{mg}$, yield 55\%). Product was isolated as oil after column chromatography on silica gel (15 g, hex/AcOEt $80: 20 \rightarrow 70: 30){ }^{1} \mathrm{H}$ NMR (400 $\left.\mathrm{MHz}, \mathrm{CDCl}_{3}\right) \delta 7.16(\mathrm{~d}, J=8.4 \mathrm{~Hz}, 2 \mathrm{H}), 6.64(\mathrm{~d}, J=8.4 \mathrm{~Hz}, 2 \mathrm{H})$, $6.57(\mathrm{t}, J=2.3 \mathrm{~Hz}, 1 \mathrm{H}), 3.75(\mathrm{~s}, 6 \mathrm{H}), 2.68(\mathrm{td}, J=7.2,2.4 \mathrm{~Hz}$, $2 \mathrm{H}), 2.36(\mathrm{t}, J=7.0 \mathrm{~Hz}, 2 \mathrm{H}), 1.82(\mathrm{p}, J=7.1 \mathrm{~Hz}, 2 \mathrm{H}) ;{ }^{13} \mathrm{C} \mathrm{NMR}$ $\left(101 \mathrm{MHz}, \mathrm{CDCl}_{3}\right) \delta 171.7,145.3,137.2,129.9,128.2,127.2$, 114.7, 65.3, 52.7, 35.8, 31.9, 24.9; IR $\left(\mathrm{CH}_{2} \mathrm{Cl}_{2}\right): 3467,3378$, 2953, 1728, 1623, 1516, 1264, 1180, 1153, 825, $526 \mathrm{~cm}^{-1}$; MS (EI): $m / z(\%)=290(21), 289(67)\left[\mathrm{M}^{+}\right], 231(29), 230(100), 229(21)$, 202(19), 171(30), 170(94), 143(21), 106(22), 73(38), 57(28), 55(17), 43(23); HRMS (EI): $m / z$ calcd for $\mathrm{C}_{16} \mathrm{H}_{19} \mathrm{NO}_{4} 289.1314$; found 289.1316 .

\section{Dimethyl (2E)-2-(4-(dimethylamino)benzylidene) cyclopentane-1,1-dicarboxylate (5)}

Prepared in reaction of dimethyl 4-pentenylmalonate and 4bromo- $N, N$-dimethylaniline following general procedure (85 mg, yield 67\%). Product was isolated as oil after column chromatography on silica gel $(15 \mathrm{~g}$, hex/AcOEt $90: 10 \rightarrow$ 70 : 30). ${ }^{1} \mathrm{H}$ NMR $\left(400 \mathrm{MHz}, \mathrm{CDCl}_{3}\right) \delta$ 7.29-7.24 (m, 2H), 6.70 (d, $J=8.8 \mathrm{~Hz}, 2 \mathrm{H}), 6.60(\mathrm{t}, J=2.3 \mathrm{~Hz}, 1 \mathrm{H}), 3.76(\mathrm{~s}, 6 \mathrm{H}), 2.96(\mathrm{~s}, 6 \mathrm{H})$, $2.72(\mathrm{td}, J=7.2,2.4 \mathrm{~Hz}, 2 \mathrm{H}), 2.38(\mathrm{t}, J=7.0 \mathrm{~Hz}, 2 \mathrm{H}), 1.83(\mathrm{p}, J=$ $7.1 \mathrm{~Hz}, 2 \mathrm{H}) ;{ }^{13} \mathrm{C}$ NMR $\left(101 \mathrm{MHz}, \mathrm{CDCl}_{3}\right) \delta 171.8,149.3,136.5$, 129.7, 127.2, 126.2, 112.1, 65.3, 52.6, 40.4, 35.8, 31.9, 24.9; IR $\left(\mathrm{CH}_{2} \mathrm{Cl}_{2}\right): 2952,2881,2804,1730,1608,1522,1434,1355,1247$, 1162, 1064, 813, $530 \mathrm{~cm}^{-1}$; MS (EI): $\mathrm{m} / z(\%)=318(21), 317(69)$ $\left[\mathrm{M}^{+}\right], 259(29), 258(100)$, 199(20), 198(49), 171(12), 153(9), 134(13), 77(5), 59(7); HRMS (EI): $\mathrm{m} / z$ calcd for $\mathrm{C}_{18} \mathrm{H}_{23} \mathrm{NO}_{4}$ 317.1627 ; found 317.1636 . 
Dimethyl (2E)-2-(4-(trifluoromethyl)benzylidene) cyclopentane-1,1-dicarboxylate (6)

Prepared in reaction of dimethyl 4-pentenylmalonate and 4-bromobenzotrifluoride following general procedure $(91 \mathrm{mg}$, yield $67 \%)$ or in reaction with 4-chlorobenzotrifluoride following modified general procedure (run at $80{ }^{\circ} \mathrm{C}$ ) (76 mg, yield 56\%). Product was isolated as oil after column chromatography on silica gel (15 g, hex/AcOEt/DCM $86: 9.5: 0.5) .{ }^{1} \mathrm{H}$ NMR $(400 \mathrm{MHz}$, $\mathrm{CDCl}_{3} \delta 7.58(\mathrm{~d}, J=8.2 \mathrm{~Hz}, 2 \mathrm{H}), 7.43(\mathrm{~d}, J=8.2 \mathrm{~Hz}, 2 \mathrm{H}), 6.74(\mathrm{~s}$, $1 \mathrm{H}), 3.78(\mathrm{~s}, 6 \mathrm{H}), 2.71(\mathrm{td}, J=7.2,2.5 \mathrm{~Hz}, 2 \mathrm{H}), 2.41(\mathrm{t}, J=7.0 \mathrm{~Hz}$, $2 \mathrm{H}), 1.85$ (p, $J=7.1 \mathrm{~Hz}, 2 \mathrm{H}) ;{ }^{13} \mathrm{C}$ NMR $\left(101 \mathrm{MHz}, \mathrm{CDCl}_{3}\right) \delta 171.1$, 143.8, 141.1, 128.8, 128.6 (q, $J=32.5 \mathrm{~Hz}), 126.3,125.0$ (q, $J=3.6$ $\mathrm{Hz}), 124.2$ (q, $J=271.8 \mathrm{~Hz}) 65.5,52.9,35.6,32.1,24.7 ;{ }^{19} \mathrm{~F}$ NMR $\left(376 \mathrm{MHz}, \mathrm{CDCl}_{3}\right) \delta-62.52 ; \mathrm{IR}\left(\mathrm{CH}_{2} \mathrm{Cl}_{2}\right): 2956,1735,1615,1435$, $1327,1265,1125,1068,830,598 \mathrm{~cm}^{-1}$; MS (EI): $m / z(\%)=343(12)$, 342(39) [ $\left.\mathrm{M}^{+}\right]$, 323(18), 310(24), 283(40), 282(61), 252(25), 251(83), 250(41), 224(27), 223(100), 159(37), 77(14), 59(38); HRMS (EI): $m / z$ calcd for $\mathrm{C}_{17} \mathrm{H}_{17} \mathrm{~F}_{3} \mathrm{O}_{4}$ 342.1079; found 342.1084.

\section{Dimethyl (2E)-2-(4-acetylbenzylidene)cyclopentane-1,1- dicarboxylate (7)}

Prepared in reaction of dimethyl 4-pentenylmalonate and 4-bromoacetophenone following general procedure (57 mg, yield 45\%) or in reaction with 4-chloroacetophenone following modified general procedure (run at $\left.80{ }^{\circ} \mathrm{C}\right)(75 \mathrm{mg}$, yield $60 \%$, isomer $E / Z$ 55 : 45). Product was isolated as oil after column chromatography on silica gel (15 g, hex/AcOEt $90: 10 \rightarrow 80: 20) .{ }^{1} \mathrm{H}$ NMR (400 $\left.\mathrm{MHz}, \mathrm{CDCl}_{3}\right): \delta 7.92-7.88(\mathrm{~m}, 2 \mathrm{H}), 7.43-7.38(\mathrm{~m}, 2 \mathrm{H}), 6.73(\mathrm{t}, J=$ $2.6 \mathrm{~Hz}, 1 \mathrm{H}), 3.76(\mathrm{~s}, 6 \mathrm{H}), 2.71(\mathrm{td}, J=7.2,2.6 \mathrm{~Hz}, 2 \mathrm{H}), 2.57(\mathrm{~s}, 3 \mathrm{H})$, $2.39(\mathrm{t}, J=6.9 \mathrm{~Hz}, 2 \mathrm{H}), 1.84(\mathrm{p}, J=7.1 \mathrm{~Hz}, 2 \mathrm{H}) ;{ }^{13} \mathrm{C}$ NMR $(101$ $\left.\mathrm{MHz}, \mathrm{CDCl}_{3}\right): \delta 197.5,171.0,144.0,142.2,135.2,128.7,128.2$, 126.6, 65.6, 52.8, 35.6, 32.2, 26.5, 24.7; IR $\left(\mathrm{CH}_{2} \mathrm{Cl}_{2}\right): 2954,1732$, $1682,1602,1435,1360,1268,590 \mathrm{~cm}^{-1}$; HRMS (ESI): $\mathrm{m} / z$ calcd for $\mathrm{C}_{18} \mathrm{H}_{20} \mathrm{O}_{5} \mathrm{Na}$ 339.1208; found: 339.1201.

\section{Dimethyl (2E)-2-(2-methoxybenzylidene)cyclopentane-1,1- dicarboxylate (8)}

Prepared in reaction of dimethyl 4-pentenylmalonate and 2bromoanisole following general procedure ( $88 \mathrm{mg}$, yield $73 \%$ ) or in reaction with 2-chloroanisole following modified general procedure (run at $80{ }^{\circ} \mathrm{C}$ ) (63 $\mathrm{mg}$, yield $51 \%$ ). Product was isolated as oil after column chromatography on silica gel ( $15 \mathrm{~g}$, hex/ AcOEt 85 : 15). ${ }^{1} \mathrm{H}$ NMR (400 $\left.\mathrm{MHz}, \mathrm{CDCl}_{3}\right) \delta 7.34$ (dd, $J=7.6$, $1.7 \mathrm{~Hz}, 1 \mathrm{H}), 7.23-7.17(\mathrm{~m}, 1 \mathrm{H}), 6.97(\mathrm{t}, J=2.6 \mathrm{~Hz}, 1 \mathrm{H}), 6.92(\mathrm{td}, J$ $=7.5,1.2 \mathrm{~Hz}, 1 \mathrm{H}), 6.85(\mathrm{dd}, J=8.3,1.1 \mathrm{~Hz}, 1 \mathrm{H}), 3.81(\mathrm{~s}, 3 \mathrm{H}), 3.78$ $(\mathrm{s}, 6 \mathrm{H}), 2.64(\mathrm{td}, J=7.2,2.6 \mathrm{~Hz}, 2 \mathrm{H}), 2.39(\mathrm{t}, J=6.9 \mathrm{~Hz}, 2 \mathrm{H}), 1.80$ $(\mathrm{p}, J=7.1 \mathrm{~Hz}, 2 \mathrm{H}) ;{ }^{13} \mathrm{C} \mathrm{NMR}\left(101 \mathrm{MHz}, \mathrm{CDCl}_{3}\right) \delta 171.5,157.0$, 140.5, 128.9, 128.1, 126.7, 122.3, 120.0, 110.5, 64.9, 55.5, 52.6, 35.7, 31.8, 24.7; IR $\left(\mathrm{CH}_{2} \mathrm{Cl}_{2}\right): 2953,2839,1732,1597,1487,1461$, 1436, 1248, 1136, $755 \mathrm{~cm}^{-1}$; HRMS (ESI): $\mathrm{m} / z$ calcd for $\mathrm{C}_{17} \mathrm{H}_{20} \mathrm{O}_{5} \mathrm{Na} 327.1208$; found 327.1203.

\section{Dimethyl (2E)-2-(naphthalen-2-ylmethylidene)cyclopentane- 1,1-dicarboxylate (9)}

Prepared in reaction of dimethyl 4-pentenylmalonate and 2bromonaphthalene following general procedure $(90 \mathrm{mg}$, yield
$69 \%$ ). Product was isolated as oil after column chromatography on silica gel (15 g, hex/AcOEt $90: 10) .{ }^{1} \mathrm{H}$ NMR (400 MHz, $\mathrm{CDCl}_{3}$ ) $\delta$ 7.84-7.79 (m, $4 \mathrm{H}), 7.52(\mathrm{dd}, J=8.5,1.4 \mathrm{~Hz}, 1 \mathrm{H}), 7.48-7.44(\mathrm{~m}$, $2 \mathrm{H}), 6.90(\mathrm{t}, J=2.3 \mathrm{~Hz}, 1 \mathrm{H}), 3.82(\mathrm{~s}, 6 \mathrm{H}), 2.84(\mathrm{td}, J=7.2,2.5 \mathrm{~Hz}$, $2 \mathrm{H}), 2.46(\mathrm{t}, J=7.0 \mathrm{~Hz}, 2 \mathrm{H}), 1.89(\mathrm{p}, J=7.0 \mathrm{~Hz}, 2 \mathrm{H}) ;{ }^{13} \mathrm{C} \mathrm{NMR}$ $\left(101 \mathrm{MHz} \mathrm{CDCl}_{3}\right) \delta 171.4,141.5,135.1,133.3,132.23,128.0$, 127.6, 127.6, 127.5, 126.8, 126.0, 125.8, 65.5, 52.8, 35.7, 32.1, 24.8; IR $\left(\mathrm{CH}_{2} \mathrm{Cl}_{2}\right): 3053,2953,2879,1732,1434,1262,1065$, 1016, 817, 748, $477 \mathrm{~cm}^{-1}$; HRMS (ESI): $m / z$ calcd for $\mathrm{C}_{20} \mathrm{H}_{20} \mathrm{O}_{4} \mathrm{Na}$ 347.1248; found 347.1259.

\section{Dimethyl (2E)-2-(pyridin-3-ylmethylidene)cyclopentane-1,1- dicarboxylate (10)}

Prepared in reaction of dimethyl 4-pentenylmalonate and 3bromopyridine following general procedure (92 mg, yield 84\%). Product was isolated as oil after column chromatography on silica gel (15 g, hex/AcOEt $80: 20 \rightarrow 70: 30) .{ }^{1} \mathrm{H}$ NMR $(400 \mathrm{MHz}$, $\left.\mathrm{CDCl}_{3}\right) \delta 8.56(\mathrm{~d}, J=2.0 \mathrm{~Hz}, 1 \mathrm{H}), 8.41(\mathrm{dd}, J=4.8,1.5 \mathrm{~Hz}, 1 \mathrm{H})$, $7.61(\mathrm{dt}, J=7.9,1.7 \mathrm{~Hz}, 1 \mathrm{H}), 7.24-7.19(\mathrm{~m}, 1 \mathrm{H}), 6.64(\mathrm{t}, J=$ $2.4 \mathrm{~Hz}, 1 \mathrm{H}), 3.75(\mathrm{~s}, 6 \mathrm{H}), 2.67(\mathrm{td}, J=7.2,2.6 \mathrm{~Hz}, 2 \mathrm{H}), 2.37(\mathrm{t}, J=$ $7.0 \mathrm{~Hz}, 2 \mathrm{H}), 1.82(\mathrm{p}, J=7.0 \mathrm{~Hz}, 2 \mathrm{H}) ;{ }^{13} \mathrm{C} \mathrm{NMR}\left(101 \mathrm{MHz}, \mathrm{CDCl}_{3}\right.$ ) $\delta$ 171.0, 150.0, 147.7, 143.6, 135.1, 133.2, 124.0, 123.0, 65.4, 52.8, 35.6, 32.0, 24.7; IR $\left(\mathrm{CH}_{2} \mathrm{Cl}_{2}\right): 3027,2953,2879,1732,1567,1434$, 1266, 1065, 1021, 804, $710 \mathrm{~cm}^{-1}$; HRMS (ESI): $\mathrm{m} / \mathrm{z}$ calcd for $\mathrm{C}_{15} \mathrm{H}_{17} \mathrm{NO}_{4}$ 276.1212; found 276.1232.

\section{Dimethyl (2E)-2-(pyridin-2-ylmethylidene)cyclopentane-1,1- dicarboxylate (11)}

Prepared in reaction of dimethyl 4-pentenylmalonate and 2bromopyridine following general procedure (64 mg, yield 58\%) or in reaction with 2-chloropyridine following modified general procedure (run at $80{ }^{\circ} \mathrm{C}$ ) (60 mg, yield 55\%). Product was isolated as oil after column chromatography on silica gel (15 g, hex/ AcOEt $80: 20 \rightarrow 70: 30) .{ }^{1} \mathrm{H}$ NMR $\left(400 \mathrm{MHz}, \mathrm{CDCl}_{3}\right) \delta 8.57$ (dd, $J$ $=4.8,1.9,0.9 \mathrm{~Hz}, 1 \mathrm{H}), 7.59(\mathrm{td}, J=7.7,1.9 \mathrm{~Hz}, 1 \mathrm{H}), 7.28-7.20$ (m, 1H), 7.07-7.02 (m, 1H), $6.74(\mathrm{t}, J=2.7 \mathrm{~Hz}, 1 \mathrm{H}), 3.74(\mathrm{~s}, 6 \mathrm{H})$, $2.94(\mathrm{td}, J=7.3,2.6 \mathrm{~Hz}, 2 \mathrm{H}), 2.37(\mathrm{t}, J=6.9 \mathrm{~Hz}, 2 \mathrm{H}), 1.82(\mathrm{p}, J=$ $7.1 \mathrm{~Hz}, 2 \mathrm{H}) ;{ }^{13} \mathrm{C} \mathrm{NMR}\left(101 \mathrm{MHz}, \mathrm{CDCl}_{3}\right) \delta 171.1,156.4,149.1$, 146.1, 135.8, 126.6, 124.07, 121.0, 65.8, 52.8, 35.6, 32.7, 24.6; IR $\left(\mathrm{CH}_{2} \mathrm{Cl}_{2}\right): 3050,2954,2280,1732,1584,1438,1433,1263,1151$, $738,747 \mathrm{~cm}^{-1}$; HRMS (ESI): $m / z$ calcd for $\mathrm{C}_{15} \mathrm{H}_{17} \mathrm{NO}_{4} 276.1236$; found 276.1223 .

\section{Dimethyl (2E)-2-(4-(hydroxymethyl)benzylidene)cyclopentane- 1,1-dicarboxylate (12)}

Prepared in reaction of dimethyl 4-pentenylmalonate and 4bromobenzyl alcohol following general procedure (72 mg, yield $60 \%$ ). Product was isolated as oil after column chromatography on silica gel (15 g, hex/AcOEt $60: 40) .{ }^{1} \mathrm{H}$ NMR (400 MHz, $\mathrm{CDCl}_{3}$ ) $\delta 7.34-7.27(\mathrm{~m}, 4 \mathrm{H}), 6.67(\mathrm{t}, J=2.5 \mathrm{~Hz}, 1 \mathrm{H}), 4.64(\mathrm{~s}, 2 \mathrm{H}), 3.75(\mathrm{~s}$, $6 \mathrm{H}), 2.68(\mathrm{td}, J=7.2,2.6 \mathrm{~Hz}, 2 \mathrm{H}), 2.37(\mathrm{t}, J=6.9 \mathrm{~Hz}, 2 \mathrm{H}), 2.11(\mathrm{~s}$, $1 \mathrm{H}), 1.82(\mathrm{p}, J=7.1 \mathrm{~Hz}, 2 \mathrm{H}) ;{ }^{13} \mathrm{C} \mathrm{NMR}\left(101 \mathrm{MHz}, \mathrm{CDCl}_{3}\right) \delta 171.4$, 141.0, 139.5, 136.9, 128.8, 127.1, 126.7, 65.4, 64.8, 52.8, 35.7, 32.0, 24.7; IR $\left(\mathrm{CH}_{2} \mathrm{Cl}_{2}\right)$ : 3426, 2953, 2877, 1730, 1435, 1265, 1163, $1013 \mathrm{~cm}^{-1}$; HRMS (ESI): $\mathrm{m} / z$ calcd for $\mathrm{C}_{17} \mathrm{H}_{20} \mathrm{O}_{5} \mathrm{Na} 327.1208$; found 327.1205. 


\section{Dimethyl (2E)-2-(4-chlorobenzylidene)cyclopentane-1,1- dicarboxylate (13)}

Prepared in reaction of dimethyl 4-pentenylmalonate and 1bromo-4-chlorobenzene following general procedure $(70 \mathrm{mg}$, $57 \%)$. Product was isolated as oil after column chromatography on silica gel (15 g, hex/AcOEt $90: 10) .{ }^{1} \mathrm{H}$ NMR $(400 \mathrm{MHz}$, $\left.\mathrm{CDCl}_{3}\right): \delta 7.31-7.23(\mathrm{~m}, 4 \mathrm{H}), 6.65(\mathrm{t}, J=2.5 \mathrm{~Hz}, 1 \mathrm{H}), 3.76(\mathrm{~s}, 6 \mathrm{H})$, $2.66(\mathrm{td}, J=7.2,2.6 \mathrm{~Hz}, 2 \mathrm{H}), 2.38(\mathrm{t}, J=6.9 \mathrm{~Hz}, 2 \mathrm{H}), 1.88-1.80$ $(\mathrm{m}, 2 \mathrm{H}) ;{ }^{13} \mathrm{C} \mathrm{NMR}\left(101 \mathrm{MHz}, \mathrm{CDCl}_{3}\right): \delta 171.2,141.7,136.0,132.5$, 129.9, 128.3 , 126.3 , 65.4 , 52.8, 35.7, 32.0, 24.7; IR $\left(\mathrm{CH}_{2} \mathrm{Cl}_{2}\right)$ : 2953, 1733, 1491, 1434, 1265, 821, $519 \mathrm{~cm}^{-1}$; HRMS (ESI): $\mathrm{m} / z$ calcd for $\mathrm{C}_{16} \mathrm{H}_{17} \mathrm{O}_{4} \mathrm{ClNa} 331.0713$; found 331.0706.

\section{Dimethyl (2E)-2-(isoquinolin-5-ylmethylidene)cyclopentane- 1,1-dicarboxylate (14)}

Prepared in reaction of dimethyl 4-pentenylmalonate and 5bromoisoquinoline following general procedure $(93 \mathrm{mg}$, yield $72 \%)$. Product was isolated as oil after column chromatography on silica gel (15 g, hex/AcOEt $70: 30 \rightarrow 60: 40) .{ }^{1} \mathrm{H}$ NMR (400 $\left.\mathrm{MHz}, \mathrm{CDCl}_{3}\right) \delta 9.21(\mathrm{~s}, 1 \mathrm{H}), 8.53(\mathrm{~d}, J=6.0 \mathrm{~Hz}, 1 \mathrm{H}), 7.82(\mathrm{dd}, J=$ 15.3, 6.9 Hz, 2H), 7.61-7.52 (m, 2H), $7.19(\mathrm{~s}, 1 \mathrm{H}), 3.82(\mathrm{~s}, 6 \mathrm{H})$, 2.49-2.42 (m, 4H), 1.75 (p, $J=7.0 \mathrm{~Hz}, 2 \mathrm{H}) ;{ }^{13} \mathrm{C}$ NMR (101 MHz, $\left.\mathrm{CDCl}_{3}\right) \delta 171.2,152.7,144.2,143.2,134.3,134.1,129.5,128.6$, $126.7,126.5,123.7,117.5,64.6,52.9,35.9,31.6,24.4$; IR $\left(\mathrm{CH}_{2} \mathrm{Cl}_{2}\right): 2953,1732,1617,1584,1434,1261,1152,832,762$, $475 \mathrm{~cm}^{-1}$; MS (EI): $m / z(\%)=326(12), 325(57)\left[\mathrm{M}^{+}\right], 275(9)$, 267(4), 234(75), 207(30), 206(100), 204(26), 156(13), 142(14), 98(2), 77(6), 43(12); HRMS (EI): $m / z$ calcd for $\mathrm{C}_{19} \mathrm{H}_{19} \mathrm{NO}_{4}$ 325.1314, found: 325.1317 .

\section{Dimethyl (2E)-2-(quinolin-5-ylmethylidene)cyclopentane-1,1- dicarboxylate (15)}

Prepared in reaction of dimethyl 4-pentenylmalonate and 5bromoquinoline following general procedure $(68 \mathrm{mg}$, yield $52 \%)$. Product was isolated as oil after column chromatography on silica gel (15 g, hex/AcOEt $70: 30 \rightarrow 60: 40) .{ }^{1} \mathrm{H}$ NMR (400 $\left.\mathrm{MHz}, \mathrm{CDCl}_{3}\right) \delta 8.88(\mathrm{dd}, J=4.2,1.6 \mathrm{~Hz}, 1 \mathrm{H}), 8.41-8.36(\mathrm{~m}, 1 \mathrm{H})$, $7.99(\mathrm{~d}, J=8.4 \mathrm{~Hz}, 1 \mathrm{H}), 7.67-7.62(\mathrm{~m}, 1 \mathrm{H}), 7.44-7.37(\mathrm{~m}, 2 \mathrm{H})$, $7.18(\mathrm{~s}, 1 \mathrm{H}), 3.81(\mathrm{~s}, 6 \mathrm{H}), 2.44(\mathrm{td}, J=7.0,2.1 \mathrm{~Hz}, 4 \mathrm{H}), 1.73(\mathrm{p}, J=$ $7.0 \mathrm{~Hz}, 2 \mathrm{H}) ;{ }^{13} \mathrm{C}$ NMR $\left(101 \mathrm{MHz}, \mathrm{CDCl}_{3}\right) \delta 171.3,150.1,148.3$, 144.1, 135.4, 133.2, 128.7, 128.6, 126.8, 126.0, 124.1, 120.9, 64.5, 52.9, 35.9, 31.5, 24.4; IR $\left(\mathrm{CH}_{2} \mathrm{Cl}_{2}\right): 2952,1731,1593,1572,1434$, 1254, 1148, $806 \mathrm{~cm}^{-1}$; MS (EI): $m / z(\%)=326(10), 325(41)\left[\mathrm{M}^{+}\right]$, 265(23), 248(20), 235(27), 234(73), 207(35), 206(100), 204(36), 152(9), 142(24), 59(11); HRMS (EI): $\mathrm{m} / \mathrm{z}$ calcd for $\mathrm{C}_{19} \mathrm{H}_{19} \mathrm{NO}_{4}$ 325.1314 ; found 325.1312 .

\section{Dimethyl (2E)-2-(1,3-benzodioxol-5-ylmethylidene) cyclopentane-1,1-dicarboxylate (16)}

Prepared in reaction of dimethyl 4-pentenylmalonate and 4bromo-1,2-methylenedioxybenzene following general procedure (112 mg, yield 88\%). Product was isolated as oil after column chromatography on silica gel $(15 \mathrm{~g}$, hex/AcOEt $70: 30$ $\rightarrow 50: 50) .{ }^{1} \mathrm{H}$ NMR (400 MHz, $\mathrm{CDCl}_{3}$ ) $\delta 6.87$ (s, 1H), 6.83-6.74 $(\mathrm{m}, 2 \mathrm{H}), 6.59(\mathrm{t}, J=2.3 \mathrm{~Hz}, 1 \mathrm{H}), 5.93(\mathrm{~s}, 2 \mathrm{H}), 3.76(\mathrm{~s}, 6 \mathrm{H}), 2.67$ $(\mathrm{td}, J=7.1,2.4 \mathrm{~Hz}, 2 \mathrm{H}), 2.37(\mathrm{t}, J=6.9 \mathrm{~Hz}, 2 \mathrm{H}), 1.83(\mathrm{p}, J=$ $7.0 \mathrm{~Hz}, 2 \mathrm{H}) ;{ }^{13} \mathrm{C} \mathrm{NMR}\left(101 \mathrm{MHz}, \mathrm{CDCl}_{3}\right) \delta 171.4,147.5,146.4$, 139.3, 131.9, 127.1, 123.0, 108.6, 108.1, 100.9, 65.3, 52.7, 35.7, 31.9, 24.8; IR $\left(\mathrm{CH}_{2} \mathrm{Cl}_{2}\right): 2963,2890,1730,1491,1442,1254,1038$, 930, $809 \mathrm{~cm}^{-1}$; MS (EI): $m / z(\%)=319(25), 318(81)\left[\mathrm{M}^{+}\right], 260(18)$, 259(63), 258(38), 231(29), 227(23), 200(31), 199(100), 169(36), 141(34), 135(30), 115(27), 77(13), 59(21); HRMS (EI): $\mathrm{m} / \mathrm{z}$ calcd for $\mathrm{C}_{17} \mathrm{H}_{18} \mathrm{O}_{6}$ 318.1103; found 318.1095.

\section{Dimethyl (2E)-2-(2-(methoxycarbonyl)benzylidene) cyclopentane-1,1-dicarboxylate (17)}

Prepared in reaction of dimethyl 4-pentenylmalonate and methyl 2-bromobenzoate following general procedure $(102 \mathrm{mg}$, yield $77 \%$ ). Product was isolated as oil after column chromatography on silica gel (15 g, hex/AcOEt $90: 10 \rightarrow 70: 30) .{ }^{1} \mathrm{H}$ NMR (400 MHz, $\mathrm{CDCl}_{3}$ ) $\delta 7.89$ (dd, $\left.J=7.8,1.4 \mathrm{~Hz}, 1 \mathrm{H}\right), 7.48-7.39$ $(\mathrm{m}, 2 \mathrm{H}), 7.30-7.25(\mathrm{~m}, 1 \mathrm{H}), 7.19(\mathrm{t}, J=2.6 \mathrm{~Hz}, 1 \mathrm{H}), 3.85(\mathrm{~s}, 3 \mathrm{H})$, $3.80(\mathrm{~s}, 6 \mathrm{H}), 2.49(\mathrm{td}, J=7.2,2.6 \mathrm{~Hz}, 2 \mathrm{H}), 2.38(\mathrm{t}, J=6.9 \mathrm{~Hz}, 2 \mathrm{H})$, 1.77 (p, $J=7.1 \mathrm{~Hz}, 2 \mathrm{H}) ;{ }^{13} \mathrm{C}$ NMR $\left(101 \mathrm{MHz}, \mathrm{CDCl}_{3}\right) \delta 171.3$, 167.5, 141.1, 138.9, 131.4, 130.3, 129.8, 129.3, 126.9, 126.7, 64.4, 52.7, 51.9, 35.8, 31.3, 24.6; IR $\left(\mathrm{CH}_{2} \mathrm{Cl}_{2}\right): 2953,1729,1598,1569$, 1434, 1257, 1127, 1078, 777, $740 \mathrm{~cm}^{-1}$; MS (EI): $\mathrm{m} / z(\%)=332(8)$ $\left[\mathrm{M}^{+}\right], 301(12), 300(24), 273(23), 268(12), 241(42), 240(100)$, 213(38), 182(27), 181(72), 153(32), 128(17), 115(18), 91(12), 77(14), 59(23); HRMS (EI): $m / z$ calcd for $\mathrm{C}_{18} \mathrm{H}_{20} \mathrm{O}_{6} 332.1260$; found 332.1255 .

\section{Dimethyl (2E)-2-(1,3-benzothiazol-5-ylmethylidene) cyclopentane-1,1-dicarboxylate (18)}

Prepared in reaction of dimethyl 4-pentenylmalonate and 5bromobenzothiazole following general procedure $(69 \mathrm{mg}$, yield $52 \%)$. Product was isolated as orange solid after column chromatography on silica gel (15 g, hex/AcOEt $75: 25) .{ }^{1} \mathrm{H}$ NMR (400 $\left.\mathrm{MHz} \mathrm{CDCl}_{3}\right) \delta 8.97(\mathrm{~s}, 1 \mathrm{H}), 8.11(\mathrm{~d}, J=1.7 \mathrm{~Hz}, 1 \mathrm{H}), 7.88(\mathrm{~d}, J=$ $8.4 \mathrm{~Hz}, 1 \mathrm{H}), 7.41(\mathrm{dd}, J=8.4,1.7 \mathrm{~Hz}, 1 \mathrm{H}), 6.85(\mathrm{t}, J=2.6 \mathrm{~Hz}, 1 \mathrm{H})$, $3.78(\mathrm{~s}, 6 \mathrm{H}), 2.78(\mathrm{td}, J=7.2,2.6 \mathrm{~Hz}, 2 \mathrm{H}), 2.41(\mathrm{t}, J=6.9 \mathrm{~Hz}, 2 \mathrm{H})$, $1.86(\mathrm{p}, J=7.0 \mathrm{~Hz}, 2 \mathrm{H}) ;{ }^{13} \mathrm{C} \mathrm{NMR}\left(101 \mathrm{MHz}, \mathrm{CDCl}_{3}\right) \delta 171.3$, 154.3, 153.6, 141.9, 136.1, 132.0, 126.8, 126.8, 123.1, 121.3, 65.4, 52.8, 35.7, 32.1, 24.8; IR $\left(\mathrm{CH}_{2} \mathrm{Cl}_{2}\right): 2952,1731,1540,1438,1264$, 1153, 1065, $849 \mathrm{~cm}^{-1}$; MS (EI): $m / z(\%)=332(21), 331(62)\left[\mathrm{M}^{+}\right]$, 272(10), 241(20), 240(62), 213(29), 212(100), 186(18), 152(14), 148(28), 59(14); HRMS (EI): $m / z$ calcd for $\mathrm{C}_{17} \mathrm{H}_{17} \mathrm{NO}_{4} \mathrm{~S}: 331.0878$; found 331.0885 .

\section{Dimethyl (2E)-2-((2-methyl-1,3-benzoxazol-5-yl)methylidene) cyclopentane-1,1-dicarboxylate(19)}

Prepared in reaction of dimethyl 4-pentenylmalonate and 5bromo-2-methyl-1,3-benzoxazole following general procedure (118 mg, yield 89\%). Product was isolated as oil after column chromatography on silica gel (15 g, hex/AcOEt $70: 30) .{ }^{1} \mathrm{H}$ NMR $\left(400 \mathrm{MHz}, \mathrm{CDCl}_{3}\right) \delta 7.61(\mathrm{~d}, J=2.0 \mathrm{~Hz}, 1 \mathrm{H}), 7.37(\mathrm{~d}, J=8.4 \mathrm{~Hz}$, $1 \mathrm{H}), 7.23(\mathrm{dd}, J=8.5,1.8 \mathrm{~Hz}, 1 \mathrm{H}), 6.77(\mathrm{t}, J=2.6 \mathrm{~Hz}, 1 \mathrm{H}), 3.75(\mathrm{~s}$, $6 \mathrm{H}), 2.70(\mathrm{td}, J=7.2,2.6 \mathrm{~Hz}, 2 \mathrm{H}), 2.59(\mathrm{~s}, 3 \mathrm{H}), 2.37(\mathrm{t}, J=6.9 \mathrm{~Hz}$, $2 \mathrm{H}), 1.82(\mathrm{p}, J=7.1 \mathrm{~Hz}, 2 \mathrm{H}) ;{ }^{13} \mathrm{C} \mathrm{NMR}\left(101 \mathrm{MHz}, \mathrm{CDCl}_{3}\right) \delta 171.3$, 164.2, 149.8, 141.6, 140.6, 134.1, 127.1, 125.9, 119.0, 109.6, 65.3, 52.7, 35.7, 31.9, 24.8, 14.4; IR $\left(\mathrm{CH}_{2} \mathrm{Cl}_{2}\right): 3456,2954,1732,1578$, 
1434, 1265, 919, $812 \mathrm{~cm}^{-1}$; MS (EI): $\mathrm{m} / z(\%)=330(15), 329(45)$ $\left[\mathrm{M}^{+}\right], 269(37), 252(23), 238(59), 211(30), 210(100), 169(33)$, 146(28), 141(31), 115(23), 91(5), 77(9), 59(19); HRMS (EI): $\mathrm{m} / \mathrm{z}$ calcd for $\mathrm{C}_{18} \mathrm{H}_{19} \mathrm{NO}_{5} 329.1263$; found 329.1274.

\section{Dimethyl (2E)-2-(4-cyanobenzylidene)cyclopentane-1,1- dicarboxylate (20)}

Prepared in reaction of dimethyl 4-pentenylmalonate and 4bromobenzonitrile following general procedure $(73 \mathrm{mg}$, yield $62 \%$ ) or in reaction with 4-chlorobenzonitrile following modified general procedure (run at $\left.80{ }^{\circ} \mathrm{C}\right)(47 \mathrm{mg}$, yield $39 \%$, isomer $E / Z 56$ : 44). Product was isolated as oil after column chromatography on silica gel (15 g, hex/AcOEt $98: 2 \rightarrow 90: 10) .{ }^{1} \mathrm{H}$ NMR (400 MHz, $\left.\mathrm{CDCl}_{3}\right) \delta 7.60(\mathrm{~d}, J=8.3 \mathrm{~Hz}, 2 \mathrm{H}), 7.41(\mathrm{~d}, J=$ $8.3 \mathrm{~Hz}, 2 \mathrm{H}), 6.71(\mathrm{t}, J=2.4 \mathrm{~Hz}, 1 \mathrm{H}), 3.76(\mathrm{~s}, 6 \mathrm{H}), 2.68(\mathrm{td}, J=7.2$, $2.5 \mathrm{~Hz}, 2 \mathrm{H}), 2.39(\mathrm{t}, J=6.9 \mathrm{~Hz}, 2 \mathrm{H}), 1.89-1.80(\mathrm{~m}, 2 \mathrm{H}) ;{ }^{13} \mathrm{C} \mathrm{NMR}$ $\left(101 \mathrm{MHz}, \mathrm{CDCl}_{3}\right) \delta 170.8,145.1,142.0,131.9,129.1,126.1$, 118.9, 110.1, 65.6, 52.9, 35.5, 32.2, 24.7; IR $\left(\mathrm{CH}_{2} \mathrm{Cl}_{2}\right): 2955,2225$, 1732, 1604, 1435, 1264, 1115, 826, $555 \mathrm{~cm}^{-1}$; HRMS (ESI): $\mathrm{m} / z$ calcd for $\mathrm{C}_{17} \mathrm{H}_{17} \mathrm{NO}_{4} \mathrm{Na} 322.1055$; found: 322.1045 .

\section{Dimethyl (2E)-2-(4-formylbenzylidene)cyclopentane-1,1- dicarboxylate (21)}

Prepared in reaction of dimethyl 4-pentenylmalonate and 4bromobenzaldehyde following general procedure (62 mg, yield $51 \%$ ). Product was isolated as oil after column chromatography on silica gel (15 g, hex/AcOEt $90: 10 \rightarrow 80: 20) .{ }^{1} \mathrm{H}$ NMR (400 $\left.\mathrm{MHz} \mathrm{CDCl}_{3}\right) \delta 9.97(\mathrm{~s}, 1 \mathrm{H}), 7.83(\mathrm{~d}, J=7.9 \mathrm{~Hz}, 2 \mathrm{H}), 7.48(\mathrm{~d}, J=$ $7.9 \mathrm{~Hz}, 2 \mathrm{H}), 6.75(\mathrm{t}, J=2.8 \mathrm{~Hz}, 1 \mathrm{H}), 3.77(\mathrm{~d}, J=1.1 \mathrm{~Hz}, 6 \mathrm{H}), 2.72$ $(\mathrm{td}, J=7.3,2.6 \mathrm{~Hz}, 2 \mathrm{H}), 2.42-2.37(\mathrm{~m}, 2 \mathrm{H}), 1.85(\mathrm{p}, J=7.1 \mathrm{~Hz}$, $2 \mathrm{H}) ;{ }^{13} \mathrm{C}$ NMR $\left(101 \mathrm{MHz}, \mathrm{CDCl}_{3}\right) \delta$ 191.7, 171.0, 144.8, 143.7, 134.6, 129.6, 129.1, 126.6, 65.6, 52.9, 35.6, 32.3, 24.8; IR $\left(\mathrm{CH}_{2} \mathrm{Cl}_{2}\right): 2954,2840,1731,1696,1602,1565,1434,1264,1168$, 822, 792, $523 \mathrm{~cm}^{-1}$; MS (EI): $m / z(\%)=303(17), 302(63)\left[\mathrm{M}^{+}\right]$, 270(41), 243(42), 242(50), 213(21), 211(99), 210(54), 183(73), 156(27), 155(100), 153(49), 128(33), 115(31), 91(37), 77(27), 59(33); HRMS (EI): $\mathrm{m} / \mathrm{z}$ calcd for $\mathrm{C}_{17} \mathrm{H}_{18} \mathrm{O}_{5}$ 302.1154; found 302.1159 .

\section{Dimethyl (2E)-2-(3-((tert-butoxycarbonyl)amino)benzylidene) cyclopentane-1,1-dicarboxylate (22)}

Prepared in reaction of dimethyl 4-pentenylmalonate and $\mathrm{N}$ (tert-butoxycarbonyl)-3-bromoaniline following general procedure (148 mg, yield 95\%). Product was isolated as oil after column chromatography on silica gel (15 g, hex/AcOEt 85 : 15 $\rightarrow 80: 20) .{ }^{1} \mathrm{H}$ NMR (400 MHz, $\mathrm{CDCl}_{3}$ ) $\delta 7.39$ (s, 1H), 7.24-7.16 $(\mathrm{m}, 2 \mathrm{H}), 7.00(\mathrm{dt}, J=7.2,1.7 \mathrm{~Hz}, 1 \mathrm{H}), 6.65(\mathrm{t}, J=2.7 \mathrm{~Hz}, 1 \mathrm{H})$, $6.60(\mathrm{~s}, 1 \mathrm{H}), 3.75(\mathrm{~s}, 6 \mathrm{H}), 2.70(\mathrm{td}, J=7.2,2.6 \mathrm{~Hz}, 2 \mathrm{H}), 2.37(\mathrm{t}, J=$ $6.9 \mathrm{~Hz}, 2 \mathrm{H}), 1.81$ (p, $J=7.1 \mathrm{~Hz}, 2 \mathrm{H}), 1.50(\mathrm{~s}, 9 \mathrm{H}) ;{ }^{13} \mathrm{C}$ NMR (101 $\left.\mathrm{MHz}, \mathrm{CDCl}_{3}\right) \delta 171.3,152.7,141.4,138.4,138.3,128.6,127.2$, 123.4, 118.7, 117.1, 80.3, 65.4, 52.7, 35.7, 32.0, 28.3, 24.8; IR $\left(\mathrm{CH}_{2} \mathrm{Cl}_{2}\right): 3361,2976,2955,1729,1538,1435,1237,1160,1065$, 888, 737, 693, $463 \mathrm{~cm}^{-1}$; HRMS (EI): $m / z$ calcd for $\mathrm{C}_{21} \mathrm{H}_{27} \mathrm{NO}_{6}$ 389.1736; found 389.1736 .
Dimethyl (2E)-2-(thiophen-2-ylmethylidene)cyclopentane-1,1dicarboxylate (23)

Prepared in reaction of dimethyl 4-pentenylmalonate and 2bromothiophene following general procedure $(53 \mathrm{mg}$, yield $47 \%)$. Product was isolated as oil after column chromatography on silica gel (15 g, hex/AcOEt $98: 2 \rightarrow 90: 10) .{ }^{1} \mathrm{H}$ NMR (400 $\left.\mathrm{MHz} \mathrm{CDCl}_{3}\right) \delta 7.31-7.27(\mathrm{~m}, 1 \mathrm{H}), 7.04-7.01(\mathrm{~m}, 2 \mathrm{H}), 6.93(\mathrm{t}, J=$ $2.7 \mathrm{~Hz}, 1 \mathrm{H}), 3.76(\mathrm{~s}, 6 \mathrm{H}), 2.69(\mathrm{td}, J=7.3,2.6 \mathrm{~Hz}, 2 \mathrm{H}), 2.40(\mathrm{t}, J=$ $6.9 \mathrm{~Hz}, 2 \mathrm{H}), 1.90(\mathrm{p}, J=7.1 \mathrm{~Hz}, 2 \mathrm{H}) ;{ }^{13} \mathrm{C} \mathrm{NMR}\left(101 \mathrm{MHz}, \mathrm{CDCl}_{3}\right)$ $\delta 171.1,141.3,138.7,127.4,126.9,125.6,120.8,65.3,52.8,36.1$, 32.2, 24.5; IR $\left(\mathrm{CH}_{2} \mathrm{Cl}_{2}\right): 2952,1731,1433,1261,1148,701 \mathrm{~cm}^{-1}$; MS (EI): $m / z(\%)=280(53)\left[\mathrm{M}^{+}\right], 222(36), 221(100), 220(84)$, 190(21), 189(62), 167(30), 161(87), 128(32), 115(20), 97(52), 77(23), 59(32); HRMS (EI): $\mathrm{m} / \mathrm{z}$ calcd for $\mathrm{C}_{14} \mathrm{H}_{16} \mathrm{O}_{4} \mathrm{~S} 280.0769$; found 280.0761 .

\section{Dimethyl (2E)-2-(2-chlorobenzylidene)cyclopentane-1,1- dicarboxylate (24)}

Prepared in reaction of dimethyl 4-pentenylmalonate and 1chloro-2-bromobenzene following general procedure $(114 \mathrm{mg}$, yield 93\%). Product was isolated as oil after column chromatography on silica gel (15 g, hex/AcOEt $90: 10) .{ }^{1} \mathrm{H}$ NMR (400 $\left.\mathrm{MHz} \mathrm{CDCl}_{3}\right) \delta$ 7.40-7.34 (m, 2H), 7.24-7.19 (m, 1H), 7.18-7.13 $(\mathrm{m}, 1 \mathrm{H}), 6.95(\mathrm{t}, J=2.6 \mathrm{~Hz}, 1 \mathrm{H}), 3.79(\mathrm{~s}, 6 \mathrm{H}), 2.58(\mathrm{td}, J=7.2$, $2.6 \mathrm{~Hz}, 2 \mathrm{H}), 2.39(\mathrm{t}, J=6.9 \mathrm{~Hz}, 2 \mathrm{H}), 1.81(\mathrm{p}, J=7.0 \mathrm{~Hz}, 2 \mathrm{H}) ;{ }^{13} \mathrm{C}$ NMR (101 MHz, $\left.\mathrm{CDCl}_{3}\right) \delta 171.1,142.9,135.7,133.7,129.6,129.3$, 128.1, 126.2 , 124.5, 64.7, 52.8, 35.7, 31.6, 24.7; IR $\left(\mathrm{CH}_{2} \mathrm{Cl}_{2}\right): 2953$, 1733, 1590, 1436, 1258, 1138, 751, $606 \mathrm{~cm}^{-1}$; MS (EI): $\mathrm{m} / z(\%)=$ 310(22), 308(48)[ $\left.\mathrm{M}^{+}\right], 276(27), 249(42), 248(48), 219(42), 217(83)$, 213(94), 191(48), 189(100), 153(65), 125(62), 115(26), 77(27); HRMS (EI) $m / z$ calcd for $\mathrm{C}_{16} \mathrm{H}_{17} \mathrm{O}_{4} \mathrm{Cl} 308.0815$; found 308.0819.

\section{Dimethyl (2E)-2-(4-nitrobenzylidene)cyclopentane-1,1- dicarboxylate (25)}

Prepared in reaction of dimethyl 4-pentenylmalonate and 4bromonitrobenzene following modified general procedure (run at $\left.80{ }^{\circ} \mathrm{C}\right)(61 \mathrm{mg}$, yield $48 \%$, isomer $E / Z 20: 80)$ or with 4 chloronitrobenzene following modified general procedure (run at $\left.80{ }^{\circ} \mathrm{C}\right)(50 \mathrm{mg}$, yield $40 \%$, isomer $E / Z 45: 55)$. Product was isolated as oil after column chromatography on silica gel (15 g, hex/AcOEt $75: 25) .{ }^{1} \mathrm{H}$ NMR (400 MHz, $\left.\mathrm{CDCl}_{3}\right) \delta 8.14-8.08(\mathrm{~m}$, $4 \mathrm{H}), 5.41-5.38(\mathrm{~m}, 1 \mathrm{H}), 3.67(\mathrm{~s}, 6 \mathrm{H}), 3.64(\mathrm{q}, J=2.3 \mathrm{~Hz}, 2 \mathrm{H})$, 2.57-2.51 (m, 2H), 2.40-2.33 (m, 2H); ${ }^{13} \mathrm{C}$ NMR (101 MHz, $\left.\mathrm{CDCl}_{3}\right) \delta 171.2,170.5,147.5,146.5,146.4,145.3,143.5,139.9$, 134.1, 123.0, 129.2, 124.9, 123.4, 122.9, 67.6, 64.0, 52.6, 52.5, $39.4,35.0,35.0,33.9,30.4,22.6$. Indicative signals of minor isomer: ${ }^{1} \mathrm{H}$ NMR (400 MHz, $\left.\mathrm{CDCl}_{3}\right) \delta$ 7.49-7.45 (m, 2H), 7.37$7.32(\mathrm{~m}, 2 \mathrm{H}), 6.67(\mathrm{t}, J=2.4 \mathrm{~Hz}, 1 \mathrm{H}), 3.47(\mathrm{~s}, 6 \mathrm{H}), 2.68(\mathrm{td}, J=$ $7.6,2.2 \mathrm{~Hz}, 2 \mathrm{H}), 2.44(\mathrm{t}, J=7.0 \mathrm{~Hz}, 2 \mathrm{H}), 1.76(\mathrm{p}, J=7.3 \mathrm{~Hz}, 2 \mathrm{H})$; MS (EI): $m / z(\%)=319(36)\left[\mathrm{M}^{+}\right], 287(33), 260(48), 259(100)$, 229(28), 228(99), 227(48), 200(73), 154(66), 128(31), 115(30), 106(14), 90(19), 77(25), 59(49), 39(16); IR $\left(\mathrm{CH}_{2} \mathrm{Cl}_{2}\right)$ : 2953, 2854, 1732, 1597, 1519, 1434, 1346, 1266, 1156, 1066, $857 \mathrm{~cm}^{-1}$; HRMS (EI): $m / z$ calcd for $\mathrm{C}_{16} \mathrm{H}_{17} \mathrm{NO}_{6} 319.1056$; found 319.1057 . 


\section{Di(propan-2-yl) (2E)-2-benzylidenecyclopentane-1,1-} dicarboxylate (26)

Prepared in reaction of dipropan-2-yl 2-pent-4ynylpropanedioate and bromobenzene following modified general procedure (run at $\left.80^{\circ} \mathrm{C}\right)(56 \mathrm{mg}$, yield $42 \%)$. Product was isolated as oil after column chromatography on silica gel (15 g, hex/AcOEt $95: 5) .{ }^{1} \mathrm{H}$ NMR (400 MHz, $\left.\mathrm{CDCl}_{3}\right) \delta 7.34-7.32(\mathrm{~m}$, $4 \mathrm{H}), 7.23-7.18(\mathrm{~m}, 1 \mathrm{H}), 6.76(\mathrm{t}, J=2.6 \mathrm{~Hz}, 1 \mathrm{H}), 5.10$ (hept, $J=$ $6.3 \mathrm{~Hz}, 2 \mathrm{H}), 2.70(\mathrm{td}, J=7.2,2.6 \mathrm{~Hz}, 2 \mathrm{H}), 2.36(\mathrm{t}, J=6.9 \mathrm{~Hz}, 2 \mathrm{H})$, $1.82(\mathrm{p}, J=7.1 \mathrm{~Hz}, 2 \mathrm{H}), 1.28(\mathrm{dd}, J=6.3,4.1 \mathrm{~Hz}, 12 \mathrm{H}) ;{ }^{13} \mathrm{C} \mathrm{NMR}$ $\left(101 \mathrm{MHz}, \mathrm{CDCl}_{3}\right) \delta 170.3,141.2,137.9,128.6,128.1,127.2$, 126.6, 68.8, 65.2, 35.6, 32.1, 24.7, 21.6, 21.5; IR $\left(\mathrm{CH}_{2} \mathrm{Cl}_{2}\right): 3450$, 2980, 2875, 1722, 1449, 1374, 1251, 1104, 909, 777, 699, $517 \mathrm{~cm}^{-1}$. MS (EI): $\mathrm{m} / z(\%)=330(27)\left[\mathrm{M}^{+}\right], 244(35), 202(59)$, 201(72), 184(54), 183(57), 173(44), 155(100), 129(43), 115(29), 91(56), 77(25), 43(95); HRMS (EI): $m / z$ calcd for $\mathrm{C}_{20} \mathrm{H}_{26} \mathrm{O}_{4}$ 330.1831 ; found 330.1822 .

\section{Di(propan-2-yl) (2E)-2-(4-methoxybenzylidene)cyclopentane- 1,1-dicarboxylate (27)}

Prepared in reaction of dipropan-2-yl 2-pent-4ynylpropanedioate and 4-bromoanisole following modified general procedure (run at $\left.80^{\circ} \mathrm{C}\right)(68 \mathrm{mg}$, yield $47 \%)$. Product was isolated as oil after column chromatography on silica gel (15 g, hex/AcOEt $90: 10) .{ }^{1} \mathrm{H}$ NMR (400 MHz, $\left.\mathrm{CDCl}_{3}\right) \delta 7.29-7.24(\mathrm{~m}$, $2 \mathrm{H}), 6.88-6.84(\mathrm{~m}, 2 \mathrm{H}), 6.68(\mathrm{t}, J=2.6 \mathrm{~Hz}, 1 \mathrm{H}), 5.08$ (hept, $J=$ $6.2 \mathrm{~Hz}, 2 \mathrm{H}), 3.80(\mathrm{~s}, 3 \mathrm{H}), 2.67(\mathrm{td}, J=7.2,2.6 \mathrm{~Hz}, 2 \mathrm{H}), 2.33(\mathrm{t}, J=$ $6.9 \mathrm{~Hz}, 2 \mathrm{H}), 1.81(\mathrm{p}, J=7.1 \mathrm{~Hz}, 2 \mathrm{H}), 1.26(\mathrm{dd}, J=6.3,4.0 \mathrm{~Hz}$, $12 \mathrm{H}) ;{ }^{13} \mathrm{C}$ NMR $\left(101 \mathrm{MHz}, \mathrm{CDCl}_{3}\right) \delta 170.4,158.3,139.0,130.7$, 129.9, 126.7, 113.6, 68.7, 65.1, 55.2, 35.6, 32.0, 24.7, 21.6, 21.5; IR $\left(\mathrm{CH}_{2} \mathrm{Cl}_{2}\right): 2979,2936,1724,1607,1511,1466,1250,1103,827$, $530 \mathrm{~cm}^{-1}$; MS (EI): $m / z(\%)=361(14), 360(43)\left[\mathrm{M}^{+}\right], 317(11)$, 273(36), 232(32), 231(100), 214(33), 213(30), 185(53), 171(12), 159(13), 135(17), 121(28), 115(21), 43(50), 41(24); HRMS (EI): $m / z$ calcd for $\mathrm{C}_{21} \mathrm{H}_{28} \mathrm{O}_{5}$ 360.1937; found 360.1936.

\section{Di(propan-2-yl) (2E)-2-(4-cyanobenzylidene)cyclopentane-1,1- dicarboxylate (28)}

Prepared in reaction of dipropan-2-yl 2-pent-4ynylpropanedioate and 4-bromobenzonitrile following modified general procedure (run at $\left.80{ }^{\circ} \mathrm{C}\right)(37 \mathrm{mg}$, yield $26 \%$, isomer $E / Z 75$ : 25). Product was isolated as oil after column chromatography on silica gel (25 g, hex/AcOEt $95: 5) .{ }^{1} \mathrm{H}$ NMR (400 $\left.\mathrm{MHz}, \mathrm{CDCl}_{3}\right) \delta 7.55-7.49(\mathrm{~m}, 4 \mathrm{H}), 6.62(\mathrm{t}, J=2.2 \mathrm{~Hz}, 1 \mathrm{H}), 4.81$ (hept, $J=6.3 \mathrm{~Hz}, 2 \mathrm{H}), 2.68(\mathrm{td}, J=7.6,2.2 \mathrm{~Hz}, 2 \mathrm{H}), 2.43(\mathrm{t}, J=$ $7.0 \mathrm{~Hz}, 2 \mathrm{H}), 1.76$ (p, $J=7.2 \mathrm{~Hz}, 2 \mathrm{H}), 1.13$ (d, $J=6.2 \mathrm{~Hz}, 6 \mathrm{H}), 1.04$ $(\mathrm{d}, J=6.3 \mathrm{~Hz}, 6 \mathrm{H}) ;{ }^{13} \mathrm{C} \mathrm{NMR}\left(101 \mathrm{MHz}, \mathrm{CDCl}_{3}\right) \delta$ 169.6, 144.7, 132.1, 131.5, 130.2, 129.3, 125.2, 110.1, 69.5, 64.3, 39.8, 35.8, 22.7, 21.4, 21.3; IR $\left(\mathrm{CH}_{2} \mathrm{Cl}_{2}\right): 2981,2937,2226,1724,1604,1375$, $1265,1102,1128,845,696,515 \mathrm{~cm}^{-1}$; Indicative signals of minor isomer $(Z):{ }^{1} \mathrm{H}$ NMR $(400 \mathrm{MHz}, \mathrm{CDCl} 3) \delta 7.59-7.55(\mathrm{~m}$, $2 \mathrm{H}), 7.35-7.30(\mathrm{~m}, 2 \mathrm{H}), 5.23$ (p, $J=2.1 \mathrm{~Hz}, 1 \mathrm{H}), 5.05$ (hept, $J=$ $6.3 \mathrm{~Hz}, 2 \mathrm{H}), 3.61-3.55(\mathrm{~m}, 2 \mathrm{H}), 2.55-2.48$ (m, 2H), 2.37-2.30 (m, $2 \mathrm{H}), 1.25(\mathrm{dd}, J=6.3,5.1 \mathrm{~Hz}, 12 \mathrm{H}) ;{ }^{13} \mathrm{C} \mathrm{NMR}\left(101 \mathrm{MHz}, \mathrm{CDCl}_{3}\right.$ ) $\delta 170.5,145.8,141.7,140.6,133.3,119.1,110.1,69.0,68.0,35.3$,
33.8, 30.3, 21.6, 21.6; MS (EI): $m / z(\%)=355(11)\left[\mathrm{M}^{+}\right], 313(7)$, 269(20), 227(54), 209(30), 180(40), 154(19), 116(29), 77(10), 57(11); HRMS (EI): $\mathrm{m} / z$ calcd for $\mathrm{C}_{21} \mathrm{H}_{25} \mathrm{NO}_{4} 355.1784$; found 355.1781 .

\section{Di-tert-butyl (2E)-2-benzylidenecyclopentane-1,1-dicarboxyl- ate (29)}

Prepared in reaction of di-tert-butyl pent-4-yn-1-ylpropanedioate and bromobenzene following modified general procedure (run at $\left.80{ }^{\circ} \mathrm{C}\right)(37 \mathrm{mg}$, yield $26 \%$ ). Product was isolated as oil after column chromatography on silica gel (25 g, hex/AcOEt $98: 2$ ). ${ }^{1} \mathrm{H}$ NMR (400 MHz, $\mathrm{CDCl}_{3}$ ) $\delta$ 7.36-7.29 (m, 4H), 7.23-7.17 (m, $1 \mathrm{H}), 6.77(\mathrm{t}, J=2.6 \mathrm{~Hz}, 1 \mathrm{H}), 2.68(\mathrm{td}, J=7.2,2.6 \mathrm{~Hz}, 2 \mathrm{H}), 2.30(\mathrm{t}, J$ $=6.9 \mathrm{~Hz}, 2 \mathrm{H}), 1.79(\mathrm{p}, J=7.0 \mathrm{~Hz}, 2 \mathrm{H}), 1.50(\mathrm{~s}, 18 \mathrm{H}) ;{ }^{13} \mathrm{C} \mathrm{NMR}$ $\left(101 \mathrm{MHz}, \mathrm{CDCl}_{3}\right) \delta 169.9,141.5,138.1,128.6,128.1,127.0$, $126.5,81.2,66.51,35.7,32.1,27.9,24.6$; MS (EI): $m / z(\%)=$ 247(3), 246(12), 202(34), 185(14), 184(21), 183(11), 155(21), 142(7), 129(14), 115(13), 106(9), 91(16), 79(16), 57(100), 41(34); IR $\left(\mathrm{CH}_{2} \mathrm{Cl}_{2}\right): 3054,2977,2930,1725,1599,1368,1270,1166$, 1128, 845, 696, $515 \mathrm{~cm}^{-1}$; HRMS (EI): $\mathrm{m} / z$ calcd for $\mathrm{C}_{22} \mathrm{H}_{30} \mathrm{O}_{4}$ 358.2144 ; found 358.2115 .

\section{Di-tert-butyl (2E)-2-(4-methoxybenzylidene)cyclopentane-1,1- dicarboxylate (30)}

Prepared in reaction of di-tert-butyl pent-4-yn-1-ylpropanedioate and 4-bromoanisole following modified general procedure (run at $\left.80{ }^{\circ} \mathrm{C}\right)(35 \mathrm{mg}$, yield $23 \%$ ). Product was isolated as oil after column chromatography on silica gel (25 g, hex/AcOEt $90: 10)$. ${ }^{1} \mathrm{H}$ NMR (400 MHz, $\mathrm{CDCl}_{3}$ ) $\delta$ 7.29-7.25 (m, 2H), 6.88-6.84 (m, $2 \mathrm{H}), 6.69(\mathrm{t}, J=2.5 \mathrm{~Hz}, 1 \mathrm{H}), 3.80(\mathrm{~s}, 3 \mathrm{H}), 2.65(\mathrm{td}, J=7.2,2.6 \mathrm{~Hz}$, $2 \mathrm{H}), 2.27(\mathrm{t}, J=6.9 \mathrm{~Hz}, 2 \mathrm{H}), 1.78(\mathrm{p}, J=7.1 \mathrm{~Hz}, 2 \mathrm{H}), 1.48(\mathrm{~s}$, $18 \mathrm{H}) ;{ }^{13} \mathrm{C} \mathrm{NMR}\left(101 \mathrm{MHz}, \mathrm{CDCl}_{3}\right) \delta 170.1,158.3,139.3,130.9$, 129.9, 126.4, 113.6, 81.2, 66.5, 55.2, 35.7, 32.1, 27.9, 27.7, 24.6; IR $\left(\mathrm{CH}_{2} \mathrm{Cl}_{2}\right)$ 3449, 2977, 2934, 1725, 1607, 1511, 1456, 1368, 1251, 1167, 1129, 1036, 848, $828 \mathrm{~cm}^{-1}$; MS (EI): $\mathrm{m} / z(\%)=388(7)$ $\left[\mathrm{M}^{+}\right], 276(22), 232(40), 231(100), 214(25), 203(11), 185(36)$, 171(12), 121(21), 115(15), 91(4), 77(8), 57(96), 43(12), 41(33); HRMS (EI): $m / z$ calcd for $\mathrm{C}_{23} \mathrm{H}_{32} \mathrm{O}_{5} 388.2250$; found 388.2242.

\section{Di-tert-butyl (2E)-2-(4-cyanobenzylidene)cyclopentane-1,1- dicarboxylate (31)}

Prepared in reaction of di-tert-butyl pent-4-yn-1-ylpropanedioate and 4-bromobenzonitrile following modified general procedure (run at $80{ }^{\circ} \mathrm{C}$ ) $(54 \mathrm{mg}$, yield $35 \%$, isomer $E / Z 35$ : 65). Product was isolated as oil after column chromatography on silica gel (25 g, hex/AcOEt $95: 5$ ). ${ }^{1} \mathrm{H}$ NMR (400 MHz, $\left.\mathrm{CDCl}_{3}\right) \delta 7.55-7.50$ $(\mathrm{m}, 4 \mathrm{H}), 6.57(\mathrm{t}, 1 \mathrm{H}), 2.65(\mathrm{td}, J=7.5,2.3 \mathrm{~Hz}, 3 \mathrm{H}), 2.37(\mathrm{t}, J=$ $6.9 \mathrm{~Hz}, 2 \mathrm{H}), 1.76-1.69(\mathrm{~m}, 2 \mathrm{H}), 1.28$ (s, 18H). ${ }^{13} \mathrm{C}$ NMR (101 $\left.\mathrm{MHz}, \mathrm{CDCl}_{3}\right) \delta 169.2,145.0,141.8,131.5,129.5,124.7,109.9$, $109.8,81.9,65.3,40.5,36.5,27.6,22.7$; indicative signals of minor isomer: ${ }^{1} \mathrm{H}$ NMR $\left(400 \mathrm{MHz}, \mathrm{CDCl}_{3}\right) \delta 7.61-7.56(\mathrm{~m}, 2 \mathrm{H})$, $7.42-7.37(\mathrm{~m}, 2 \mathrm{H}), 6.76(\mathrm{t}, J=2.6 \mathrm{~Hz}, 1 \mathrm{H}), 2.29(\mathrm{t}, J=6.9 \mathrm{~Hz}, 2 \mathrm{H})$, 1.84-1.76 (m, 2H), 1.47 (s, 18H); ${ }^{13} \mathrm{C} \mathrm{NMR} \mathrm{(101} \mathrm{MHz,} \mathrm{CDCl}_{3}$ ) $\delta$ 169.36, 145.66, 142.49, 131.90, 129.00, 125.56, 119.06, 81.62, 66.83, 35.46, 32.37, 27.84, 24.48; IR $\left(\mathrm{CH}_{2} \mathrm{Cl}_{2}\right): 3434,2978,2933$, 2226, 1724, 1604, 1456, 1368, 1128, 1065, 844, $555 \mathrm{~cm}^{-1}$; MS 
(EI): $m / z(\%)=384(1)\left[\mathrm{M}^{+}\right], 327(4), 283(10), 271(13), 254(9)$, 227(42), 210(15), 180(13), 153(11), 116(12), 77(3), 57(100), 43(6), 41(23).

\section{tert-Butyl (2E)-2-benzylidene-1-cyanocyclopentanecarboxylate} (32)

Prepared in reaction of tert-butyl 2-cyanohept-6-ynoate and bromobenzene following general procedure (82 mg, yield 73\%). Product was isolated as oil after column chromatography on silica gel (15 g, hex/AcOEt $90: 10) .{ }^{1} \mathrm{H}$ NMR (400 $\mathrm{MHz}, \mathrm{CDCl}_{3}$ ) $\delta$ 7.39-7.31 (m, 4H), 7.29-7.24 (m, 1H), $6.85(\mathrm{t}, J=2.6 \mathrm{~Hz}, 1 \mathrm{H})$, 2.78-2.71 (m, 2H), 2.59-2.50 (m, 1H), 2.32-2.24 (m, 1H), 2.14$2.03(\mathrm{~m}, 1 \mathrm{H}), 2.01-1.90(\mathrm{~m}, 1 \mathrm{H}), 1.52(\mathrm{~s}, 9 \mathrm{H}) ;{ }^{13} \mathrm{C}$ NMR $(101 \mathrm{MHz}$, $\left.\mathrm{CDCl}_{3}\right) \delta 166.7,141.3,136.4,128.6,128.5,128.3,127.5,127.1$, 120.1, 83.8, 53.6, 36.4, 30.8, 27.7, 25.1; MS (EI): $m / z(\%)=283(1)$ $\left[\mathrm{M}^{+}\right], 182(37), 153(10), 128(12), 115(15), 102(5), 91(17), 77(12)$, 57(100), 43(12), 41(26); IR $\left(\mathrm{CH}_{2} \mathrm{Cl}_{2}\right): 3447,2978,2877,2240$, 2214, 1737, 1449, 1370, 1256, 1150, 840, 695, $513 \mathrm{~cm}^{-1}$; HRMS (EI): $m / z$ calcd for $\mathrm{C}_{18} \mathrm{H}_{21} \mathrm{NO}_{2} 283.1572$; found 283.1564.

\section{tert-Butyl (2E)-1-cyano-2-(4-methoxybenzylidene) cyclopentanecarboxylate (33)}

Prepared in reaction of tert-butyl 2-cyanohept-6-ynoate and 4bromoanisole following modified general procedure (run at 80 $\left.{ }^{\circ} \mathrm{C}\right)(121 \mathrm{mg}$, yield 96\%). Product was isolated as oil after column chromatography on silica gel (15 g, hex/AcOEt $80: 20) .{ }^{1} \mathrm{H}$ NMR $\left(400 \mathrm{MHz}, \mathrm{CDCl}_{3}\right) \delta 7.32-7.23(\mathrm{~m}, 2 \mathrm{H}), 6.93-6.85(\mathrm{~m}, 2 \mathrm{H}), 6.77(\mathrm{t}$, $J=2.6 \mathrm{~Hz}, 1 \mathrm{H}), 3.80(\mathrm{~s}, 3 \mathrm{H}), 2.78-2.64(\mathrm{~m}, 2 \mathrm{H}), 2.58-2.46(\mathrm{~m}$, $1 \mathrm{H}), 2.31-2.20(\mathrm{~m}, 1 \mathrm{H}), 2.13-2.01(\mathrm{~m}, 1 \mathrm{H}), 2.00-1.88(\mathrm{~m}, 1 \mathrm{H})$, 1.51 (s, 9H); ${ }^{13} \mathrm{C}$ NMR (101 MHz, $\left.\mathrm{CDCl}_{3}\right) \delta 166.9,158.9,138.9$, 129.9, 129.2, 126.6, 120.3, 113.8, 83.7, 55.17, 53.60, 36.5, 30.7, 27.7, 25.1; IR $\left(\mathrm{CH}_{2} \mathrm{Cl}_{2}\right): 2978,2935,2838,2240,1735,1607,1512$, 1462, 1370, 1253, 1152, 1034, 836, $513 \mathrm{~cm}^{-1}$; MS (EI): $\mathrm{m} / z(\%)=$ 313(10) $\left[\mathrm{M}^{+}\right]$, 213(65), 212(75), 198(28), 167(32), 121(16), 115(14), 91(8), 77(13), 57(100), 43(11), 41(27); HRMS (EI): $\mathrm{m} / \mathrm{z}$ calcd for $\mathrm{C}_{19} \mathrm{H}_{23} \mathrm{NO}_{3}$ 313.1678; found 313.1680.

\section{tert-Butyl (2E)-1-cyano-2-(4-cyanobenzylidene)cyclopentane- carboxylate (34)}

Prepared in reaction of tert-butyl 2-cyanohept-6-ynoate and 4bromobenzonitrile following modified general procedure (run at $80{ }^{\circ} \mathrm{C}$ ) (40 mg, yield $33 \%$, isomer $\left.E / Z 23: 77\right)$. Product was isolated as oil after column chromatography on silica gel (15 g, hex/AcOEt $90: 10) .{ }^{1} \mathrm{H}$ NMR $\left(400 \mathrm{MHz}, \mathrm{CDCl}_{3}\right) \delta 7.61-7.56(\mathrm{~m}$, $2 \mathrm{H}), 7.35-7.29$ (m, 2H), 5.49-5.45 (m, 1H), 3.58 (dq, $J=16.6$, $2.1 \mathrm{~Hz}, 1 \mathrm{H}), 3.46(\mathrm{dq}, J=16.5,2.2 \mathrm{~Hz}, 1 \mathrm{H}), 2.66-2.57(\mathrm{~m}, 1 \mathrm{H})$, 2.54-2.44 (m, 3H), 1.45 (s, 9H); ${ }^{13} \mathrm{C} \mathrm{NMR} \mathrm{(101} \mathrm{MHz,} \mathrm{CDCl}_{3}$ ) $\delta$ 166.7, 143.3, 138.5, 134.0, 132.2, 132.1, 130.1, 129.0, 110.6, 84.1, 56.7, 36.1, 34.61, 31.0, 27.6; Indicative signals of minor isomer $(Z):{ }^{1} \mathrm{H}$ NMR (400 MHz, $\left.\mathrm{CDCl}_{3}\right) \delta 7.66-7.60(\mathrm{~m}, 2 \mathrm{H}), 7.41$ $(\mathrm{d}, J=8.3 \mathrm{~Hz}, 2 \mathrm{H}), 6.82(\mathrm{t}, J=2.7 \mathrm{~Hz}, 1 \mathrm{H}), 2.73(\mathrm{td}, J=7.3$, $2.6 \mathrm{~Hz}, 2 \mathrm{H}), 2.32-2.24(\mathrm{~m}, 1 \mathrm{H}), 2.14-2.03(\mathrm{~m}, 1 \mathrm{H}), 2.02-1.91(\mathrm{~m}$, 1H), 1.50 (s, 9H); ${ }^{13} \mathrm{C}$ NMR (101 MHz, $\left.\mathrm{CDCl}_{3}\right) \delta$ 166.2, 145.3, $140.7,119.6,118.5,110.9,84.3,53.9,36.4,25.0 ;$ IR $\left(\mathrm{CH}_{2} \mathrm{Cl}_{2}\right)$ : 3059, 2879, 2935, 2228, 1736, 1606, 1370, 1254, $1152 \mathrm{~cm}^{-1}$; MS (EI): $m / z(\%)=308(1)\left[\mathrm{M}^{+}\right], 252(10), 208(26), 207(30), 153(6)$,
116(28), 77(8), 57(100), 43(16), 41(28); HRMS (ESI): $m / z$ calcd for $\mathrm{C}_{19} \mathrm{H}_{20} \mathrm{~N}_{2} \mathrm{O}_{2} \mathrm{Na} 331.1525$; found 331.1397.

\section{Propan-2-yl (2E)-2-benzylidene-1-cyanocyclopentane- carboxylate (35)}

Prepared in reaction of propan-2-yl 2-cyanohept-6-ynoate and bromobenzene following modified general procedure (run for 4 h) (80 mg, yield 75\%). Product was isolated as oil after column chromatography on silica gel (15 g, hex/AcOEt $95: 5) .{ }^{1} \mathrm{H}$ NMR $\left(400 \mathrm{MHz}, \mathrm{CDCl}_{3}\right) \delta 7.39-7.30(\mathrm{~m}, 4 \mathrm{H}), 7.29-7.24(\mathrm{~m}, 1 \mathrm{H}), 6.84(\mathrm{t}$, $J=2.6 \mathrm{~Hz}, 1 \mathrm{H}), 5.10$ (hept, $J=6.3 \mathrm{~Hz}, 1 \mathrm{H}), 2.82-2.68(\mathrm{~m}, 2 \mathrm{H})$, 2.61-2.53 (m, 1H), 2.35-2.26 (m, 1H), 2.16-2.05 (m, 1H), 2.03$1.91(\mathrm{~m}, 1 \mathrm{H}), 1.32(\mathrm{t}, J=6.5 \mathrm{~Hz}, 6 \mathrm{H}) ;{ }^{13} \mathrm{C} \mathrm{NMR}\left(101 \mathrm{MHz}, \mathrm{CDCl}_{3}\right)$ $\delta 167.4,141.0,136.3,128.6,128.4,127.6,127.4,119.9,70.9,52.9$, 36.6, 30.8, 25.1, 21.4, 21.4; IR $\left(\mathrm{CH}_{2} \mathrm{Cl}_{2}\right): 2981,2241,1737,1450$, 1376, 1237, 1103, 762, 695, $513 \mathrm{~cm}^{-1}$; MS (EI): $m / z(\%)=270(3)$, 269(14), 184(5), 183(62), 182(82), 155(5), 129(22), 115(20), 102(8), 91(24), 77(18), 52(13), 43(100); HRMS (EI) $\mathrm{m} / \mathrm{z}$ calcd for $\mathrm{C}_{17} \mathrm{H}_{19} \mathrm{NO}_{2}$ 269.1416; found 269.1422.

\section{Propan-2-yl (2E)-1-cyano-2-(4-methoxybenzylidene) cyclopentanecarboxylate (36)}

Prepared in reaction of propan-2-yl 2-cyanohept-6-ynoate and 4bromoanisole following modified general procedure (run for 4 h) (86 mg, yield $72 \%$ ). Product was isolated as oil after column chromatography on silica gel (15 g, hex/AcOEt $90: 10) .{ }^{1} \mathrm{H}$ NMR $\left(400 \mathrm{MHz}, \mathrm{CDCl}_{3}\right) \delta 7.31-7.23(\mathrm{~m}, 2 \mathrm{H}), 6.92-6.84(\mathrm{~m}, 2 \mathrm{H}), 6.76(\mathrm{t}$, $J=2.6 \mathrm{~Hz}, 1 \mathrm{H}$ ), 5.08 (hept, $J=6.3 \mathrm{~Hz}, 1 \mathrm{H}), 3.80$ (s, 3H), 2.80$2.64(\mathrm{~m}, 2 \mathrm{H}), 2.59-2.49(\mathrm{~m}, 1 \mathrm{H}), 2.33-2.22(\mathrm{~m}, 1 \mathrm{H}), 2.15-2.04$ $(\mathrm{m}, 1 \mathrm{H}), 2.01-1.89(\mathrm{~m}, 1 \mathrm{H}), 1.31(\mathrm{t}, J=6.3 \mathrm{~Hz}, 6 \mathrm{H}) ;{ }^{13} \mathrm{C} \mathrm{NMR}(101$ $\left.\mathrm{MHz}, \mathrm{CDCl}_{3}\right) \delta 167.5,159.0,138.6,129.9,129.0,126.8,120.0$, 113.8, 70.7, 55.2, 52.9, 36.5, 30.7, 25.1, 21.4, 21.3. IR $\left(\mathrm{CH}_{2} \mathrm{Cl}_{2}\right)$ : 2981, 2937, 2240, 1736, 1606, 1512, 1465, 1253, 1178, 1103, 1034, 831, $531 \mathrm{~cm}^{-1}$; MS (EI): $\mathrm{m} / z(\%)=299(19)\left[\mathrm{M}^{+}\right], 256(4)$, 212(100), 198(10), 170(12), 121(13), 115(12), 91(6), 77(10), 43(52); HRMS (EI): $\mathrm{m} / z$ calcd for $\mathrm{C}_{18} \mathrm{H}_{21} \mathrm{NO}_{3} 299.1521$; found 299.1527.

\section{Propan-2-yl (2E)-1-cyano-2-(4-cyanobenzylidene) cyclopentanecarboxylate (37)}

Prepared in reaction of propan-2-yl 2-cyanohept-6-ynoate and 4bromobenzonitrile following modified general procedure (run for $4 \mathrm{~h}$ ) (83 mg, yield 70\%, isomer $E / Z 20: 80)$. Product was isolated as oil after column chromatography on silica gel (15 g, hex/AcOEt $90: 10) .{ }^{1} \mathrm{H}$ NMR (400 MHz, $\left.\mathrm{CDCl}_{3}\right) \delta 7.60-7.55(\mathrm{~m}$, $2 \mathrm{H}), 7.32-7.27$ (m, 2H), 5.50 (p, $J=1.9 \mathrm{~Hz}, 1 \mathrm{H}), 4.95$ (hept, $J=$ $6.3 \mathrm{~Hz}, 1 \mathrm{H}), 3.61-3.52(\mathrm{~m}, 1 \mathrm{H}), 3.48-3.40(\mathrm{~m}, 1 \mathrm{H}), 2.67-2.58(\mathrm{~m}$, 1H), 2.56-2.46 (m, 2H), 1.26-1.21 (m, 6H); ${ }^{13} \mathrm{C}$ NMR (101 MHz, $\left.\mathrm{CDCl}_{3}\right) \delta 167.3,143.1,138.2,134.3,132.7,132.4,132.2,132.1$, 130.0, 129.4, 129.0, 127.8, 118.6, 118.5, 110.6, 71.0, 56.0, 36.1, $34.5,31.0,21.4,21.3,21.3,21.3$; indicative signals of minor isomer $(Z):{ }^{1} \mathrm{H}$ NMR $\left(400 \mathrm{MHz}, \mathrm{CDCl}_{3}\right) \delta 7.78-7.72(\mathrm{~m}, 1 \mathrm{H}), 7.71-$ $7.65(\mathrm{~m}, 1 \mathrm{H}), 7.65-7.57(\mathrm{~m}, 1 \mathrm{H}), 7.40(\mathrm{~d}, J=8.4 \mathrm{~Hz}, 1 \mathrm{H}), 6.81(\mathrm{t}, J$ $=2.7 \mathrm{~Hz}, 1 \mathrm{H}), 5.08(\mathrm{p}, J=6.2 \mathrm{~Hz}, 1 \mathrm{H}), 2.77-2.70(\mathrm{~m}, 1 \mathrm{H}), 2.36-$ $2.26(\mathrm{~m}, 2 \mathrm{H}), 2.16-2.05(\mathrm{~m}, 1 \mathrm{H}), 2.03-1.95(\mathrm{~m}, 1 \mathrm{H}), 1.95-1.88$ (m, 1H), 1.32-1.27 (m, 6H); ${ }^{13} \mathrm{C}$ NMR (101 MHz, $\left.\mathrm{CDCl}_{3}\right) \delta 166.8$, 
$160.8,145.0,143.3,142.5,140.6,125.8,119.3,118.2,112.2$, $110.9,110.81,110.2,53.2,37.1,36.4,35.5,34.1,25.0,22.3$; IR $\left(\mathrm{CH}_{2} \mathrm{Cl}_{2}\right): 3452,3060,2983,2938,2228,1738,1606,1326,1248$, 1178, 1104, 834, $553 \mathrm{~cm}^{-1}$; MS (EI): $m / z(\%)=294(7)\left[\mathrm{M}^{+}\right]$, 252(10), 208(63), 204(34), 180(18), 153(15), 140(19), 116(45), 104(16), 89(20), 77(14), 43(100); HRMS (EI): $\mathrm{m} / \mathrm{z}$ calcd for $\mathrm{C}_{18} \mathrm{H}_{18} \mathrm{~N}_{2} \mathrm{O}_{2}$ 294.1368; found 294.1363.

\section{(2E)-2-Benzylidenecyclopentane-1,1-dicarbonitrile (38)}

Prepared in reaction of pent-4-yn-1-ylpropanedinitrile and bromobenzene following modified general procedure (run for $4 \mathrm{~h}$ ) (72 mg, yield 87\%). Product was isolated as oil after column chromatography on silica gel (15 g, hex/AcOEt $90: 10) .{ }^{1} \mathrm{H}$ NMR $\left(400 \mathrm{MHz}, \mathrm{CDCl}_{3}\right) \delta 7.44-7.31(\mathrm{~m}, 5 \mathrm{H}), 6.99(\mathrm{t}, J=2.7 \mathrm{~Hz}, 1 \mathrm{H})$, $2.83(\mathrm{td}, J=7.3,2.7 \mathrm{~Hz}, 2 \mathrm{H}), 2.50(\mathrm{t}, J=6.9 \mathrm{~Hz}, 2 \mathrm{H}), 2.15(\mathrm{p}, J=$ $7.1 \mathrm{~Hz}, 2 \mathrm{H}) ;{ }^{13} \mathrm{C}$ NMR (101 MHz, $\left.\mathrm{CDCl}_{3}\right) \delta 136.5,135.0,129.9$, $128.8,128.7,128.6,128.4,115.4,40.3,38.8,29.3$, 24.3; IR $\left(\mathrm{CH}_{2} \mathrm{Cl}_{2}\right): 3058,3029,2953,2246,1492,1449,1194,921,760$, 694, $512 \mathrm{~cm}^{-1}$; MS (EI): $\mathrm{m} / z(\%)=208(100)\left[\mathrm{M}^{+}\right], 207(55)$, 180(47), 153(30), 115(69), 102(21), 91(33), 77(26), 51(28), 39(26); HRMS (EI): $m / z$ calcd for $\mathrm{C}_{14} \mathrm{H}_{12} \mathrm{~N}_{2}$ 208.1000; found 208.1006.

\section{(2E)-2-(4-Methoxybenzylidene)cyclopentane-1,1-dicarbo- nitrile (39)}

Prepared in reaction of pent-4-yn-1-ylpropanedinitrile and 4bromoanisole following modified general procedure (run for $4 \mathrm{~h}$ ) (86 mg, yield 91\%). Product was isolated as oil after column chromatography on silica gel (15 g, hex/AcOEt $80: 20) .{ }^{1} \mathrm{H}$ NMR (400 MHz, $\left.\mathrm{CDCl}_{3}\right) \delta 7.34-7.28(\mathrm{~m}, 2 \mathrm{H})$, 6.95-6.89 (m, 3H), $3.83(\mathrm{~s}, 3 \mathrm{H}), 2.79(\mathrm{td}, J=7.3,2.7 \mathrm{~Hz}, 2 \mathrm{H})$, $2.47(\mathrm{t}, J=6.9 \mathrm{~Hz}, 2 \mathrm{H}), 2.13(\mathrm{p}, J=7.1 \mathrm{~Hz}, 2 \mathrm{H}) ;{ }^{13} \mathrm{C} \mathrm{NMR}(101$ $\left.\mathrm{MHz}, \mathrm{CDCl}_{3}\right) \delta 159.6,133.8,130.2,129.3,127.7,115.6,114.0$, 55.2, 40.3, 38.8, 29.2, 24.4; IR $\left(\mathrm{CH}_{2} \mathrm{Cl}_{2}\right): 2956,2839,2246$, 1606, 1513, 1463, 1254, 1179, 1032, 890, 829, $531 \mathrm{~cm}^{-1}$; MS $(\mathrm{EI}): m / z(\%)=239(29), 238(100)\left[\mathrm{M}^{+}\right], 237(27), 223(16)$, 210(29), 195(21), 170(19), 160(40), 145(40), 129(25), 115(27), 91(17), 77(20), 51(17), 43(13), 39(18); HRMS (EI): $m / z$ calcd for $\mathrm{C}_{15} \mathrm{H}_{14} \mathrm{~N}_{2} \mathrm{O}$ 238.1106; found 238.1111.

\section{(E)-Methyl 1-acetyl-2-benzylidenecyclopentanecarboxylate (40)}

Prepared in reaction of methyl 2-acetylhept-6-ynoate and bromobenzene following modified general procedure (run for $2 \mathrm{~h}$ ) (yield: $80 \%$ ) product was isolated as oil after column chromatography on silica gel (15 g, 95:5 $\rightarrow$ 90:10 hexanes/ EtOAc). ${ }^{1} \mathrm{H}$ NMR (400 MHz, $\left.\mathrm{CDCl}_{3}\right) \delta$ 7.37-7.32 (m, 4H), 7.27$7.20(\mathrm{~m}, 1 \mathrm{H}), 6.60(\mathrm{t}, J=2.5 \mathrm{~Hz}, 1 \mathrm{H}), 3.78(\mathrm{~s}, 3 \mathrm{H}), 2.80-2.63(\mathrm{~m}$, $2 \mathrm{H}), 2.50-2.41(\mathrm{~m}, 1 \mathrm{H}), 2.26(\mathrm{~s}, 3 \mathrm{H}), 2.25-2.16(\mathrm{~m}, 1 \mathrm{H}), 1.89-$ $1.75(\mathrm{~m}, 2 \mathrm{H}) ;{ }^{13} \mathrm{C} \mathrm{NMR}\left(101 \mathrm{MHz}, \mathrm{CDCl}_{3}\right) \delta 204.0,171.8,141.5$, 137.4, 128.6, 128.2, 127.6, 126.9, 72.2, 52.6, 34.4, 32.0, 26.8, 24.8; IR $\left(\mathrm{CH}_{2} \mathrm{Cl}_{2}\right): 3410,2953,1737,1714,1493,1447,1433$, 1356, 1238, $697 \mathrm{~cm}^{-1}$; MS (EI), $m / z(\%): 258$ (7, $\left.\mathrm{M}^{+}\right), 216$ (80), 184 (100), 167 (13), 155 (86), 141 (19), 128 (34), 115 (29), 105 (14), 91 (35), 77 (23), 43 (46); HRMS (EI): $\mathrm{m} / \mathrm{z}$ calcd for $\mathrm{C}_{16} \mathrm{H}_{18} \mathrm{O}_{3}$ : 258.1256. Found 258.1255.

\section{(E)-Methyl 1-acetyl-2-(4-methoxybenzylidene)cyclopentane-} carboxylate (41)

Prepared in reaction of methyl 2-acetylhept-6-ynoate and 4bromoanisole following modified general procedure (run for 2 h) (yield: 79\%). Product was isolated as oil after column chromatography on silica gel (15 g column, 90:10 $\rightarrow$ 80:20 hexanes/EtOAc). ${ }^{1} \mathrm{H}$ NMR (400 MHz, $\left.\mathrm{CDCl}_{3}\right) \delta 7.33-7.26(\mathrm{~m}, J=$ $8.7 \mathrm{~Hz}, 2 \mathrm{H}), 6.91-6.85(\mathrm{~m}, 2 \mathrm{H}), 6.53(\mathrm{t}, J=2.4 \mathrm{~Hz}, 1 \mathrm{H}), 3.81(\mathrm{~s}$, $3 \mathrm{H}), 3.77(\mathrm{~s}, 3 \mathrm{H}), 2.79-2.59(\mathrm{~m}, 2 \mathrm{H}), 2.44(\mathrm{dt}, J=13.5,6.9 \mathrm{~Hz}$, $1 \mathrm{H}), 2.25$ (s, 3H), 2.24-2.13 (m, 1H), 1.90-1.76 (m, 2H); ${ }^{13} \mathrm{C} \mathrm{NMR}$ $\left(101 \mathrm{MHz}, \mathrm{CDCl}_{3}\right) \delta$ 204.3, 172.0, 158.5, 139.2, 130.2, 129.9, 127.1, 113.7, 72.2, 55.2, 52.6, 34.4, 31.9, 26.7, 24.8; IR $\left(\mathrm{CH}_{2} \mathrm{Cl}_{2}\right)$ : 2954, 1737, 1712, 1606, 1512, 1461, 1435, 1355, 1251, 1177, 1034, 826, $531 \mathrm{~cm}^{-1}$; MS (EI), $m / z(\%): 288$ (24, $\mathrm{M}^{+}$), 245 (79), 229 (16), 214 (50), 185 (100), 171 (16), 159 (14), 141 (13), 128 (14), 121 (24), 115 (23), 77 (10), 43 (32); HRMS (EI): $\mathrm{m} / \mathrm{z}$ calcd for $\mathrm{C}_{17} \mathrm{H}_{20} \mathrm{O}_{4}: 288.1362$. Found 288.1364.

\section{(E)-Methyl 1-acetyl-2-(4-cyanobenzylidene)cyclopentane-} carboxylate (42)

Prepared in reaction of methyl 2-acetylhept-6-ynoate and 4bromobenzonitrile following modified general procedure (run for $2 \mathrm{~h}$ ) (yield: 75\%, $E / Z 91: 9)$ Product was isolated as oil after column chromatography on silica gel (15 g column, $9: 1 \rightarrow 8: 2$ hexanes/EtOAc). ${ }^{1} \mathrm{H}$ NMR $\left(400 \mathrm{MHz}, \mathrm{CDCl}_{3}\right) \delta 7.63-7.56(\mathrm{~m}, 2 \mathrm{H})$, $7.40(\mathrm{~d}, J=8.3 \mathrm{~Hz}, 2 \mathrm{H}), 6.57(\mathrm{t}, J=2.5 \mathrm{~Hz}, 1 \mathrm{H}), 3.78(\mathrm{~s}, 3 \mathrm{H}), 2.76-$ $2.59(\mathrm{~m}, 2 \mathrm{H}), 2.53-2.43(\mathrm{~m}, 1 \mathrm{H}), 2.24(\mathrm{~s}, 3 \mathrm{H}), 2.22-2.14(\mathrm{~m}, 1 \mathrm{H})$, 1.91-1.75 (m, 2H); ${ }^{13} \mathrm{C}$ NMR (101 MHz, $\left.\mathrm{CDCl}_{3}\right) \delta 202.9,171.3$, 145.4, 141.9, 132.0, 129.1, 126.2, 118.9, 110.2, 72.4, 52.9, 34.3, $32.3,26.8,24.7$; indicative signals of $Z$ isomer: ${ }^{1} \mathrm{H}$ NMR (400 $\left.\mathrm{MHz} \mathrm{CDCl}_{3}\right) \delta 7.52-7.48(\mathrm{~m}, 2 \mathrm{H}), 7.28(\mathrm{~d}, J=8.1 \mathrm{~Hz}, 2 \mathrm{H}), 6.68$ (s, 1H), $3.40(\mathrm{~s}, 3 \mathrm{H}), 2.06(\mathrm{~s}, 3 \mathrm{H}) ;{ }^{13} \mathrm{C} \mathrm{NMR}\left(101 \mathrm{MHz}, \mathrm{CDCl}_{3}\right)$ $\delta$ 204.3, 145.2, 141.3, 131.6, 125.4, 110.5, 70.3, 52.3, 38.4, 35.8, 23.1; IR $\left(\mathrm{CH}_{2} \mathrm{Cl}_{2}\right): 2954,2880,2842,2226,1738,1713,1604$, 1503, 1433, 1357, 1239, 1177, 1153, 1129, 886, 827, 555; HRMS (ESI): $m / z$ calcd for $\mathrm{C}_{17} \mathrm{H}_{17} \mathrm{NO}_{3} \mathrm{Na}\left([\mathrm{M}+\mathrm{Na}]^{+}\right)$: 306.1106. Found 306.1107.

\section{1-((2E)-1-Benzoyl-2-benzylidenecyclopentyl)ethanone (43)}

Prepared in reaction of 2-(pent-4-ynyl)-1-phenylbutane-1,3dione and bromobenzene following modified general procedure (run at $\left.80{ }^{\circ} \mathrm{C}\right)(55 \mathrm{mg}$, yield $45 \%)$. Product was isolated as oil after column chromatography on silica gel (15 g, hex/AcOEt 90 : 10). ${ }^{1} \mathrm{H}$ NMR (400 MHz, $\left.\mathrm{CDCl}_{3}\right) \delta 7.83-7.78(\mathrm{~m}, 2 \mathrm{H}), 7.54-$ 7.49 (m, 1H), 7.44-7.39 (m, 2H), 7.36-7.31 (m, 4H), 7.26-7.21 (m, 1H), 2.90-2.80 (m, 2H), 2.79-2.69 (m, 1H), 2.34 (s, 3H), 2.32$2.24(\mathrm{~m}, 1 \mathrm{H}), 1.92-1.84(\mathrm{~m}, 2 \mathrm{H}) ;{ }^{13} \mathrm{C} \mathrm{NMR}\left(101 \mathrm{MHz}, \mathrm{CDCl}_{3}\right)$ $\delta 204.8,199.2,142.2,137.4,135.5,132.5,129.3,128.8,128.6$, 128.3, 128.2, 127.0, 77.2, 34.9, 31.8, 27.4, 24.8; IR $\left(\mathrm{CH}_{2} \mathrm{Cl}_{2}\right): 3056$, 3025, 2959, 2876, 1683, 1597, 1446, 1258, 1231, 735, 696, $516 \mathrm{~cm}^{-1} ; \operatorname{MS}(\mathrm{EI}): m / z(\%)=304(5)\left[\mathrm{M}^{+}\right], 262(13), 233(12)$, 199(11), 182(13), 155(10), 128(15), 105(100), 91(19), 77(48), 51(15), 43(32); HRMS (EI) $\mathrm{m} / z$ calcd for $\mathrm{C}_{21} \mathrm{H}_{20} \mathrm{O}_{2} 304.1463$; found 304.1462 . 
1-((2E)-1-Benzoyl-2-(4-methoxybenzylidene)cyclopentyl) ethanone (44)

Prepared in reaction of 2-(pent-4-ynyl)-1-phenylbutane-1,3dione and 4-bromoanisole following modified general procedure (run at $\left.80{ }^{\circ} \mathrm{C}\right)(78 \mathrm{mg}$, yield $60 \%)$. Product was isolated as oil after column chromatography on silica gel $(15 \mathrm{~g}$, hex/AcOEt 80 : 20). ${ }^{1} \mathrm{H}$ NMR $\left(400 \mathrm{MHz}, \mathrm{CDCl}_{3}\right) \delta 7.81-7.76(\mathrm{~m}, 2 \mathrm{H}), 7.52-$ 7.47 (m, 1H), 7.42-7.36 (m, 2H), 7.29-7.24 (m, 2H), 6.90-6.85 $(\mathrm{m}, 2 \mathrm{H}), 6.40(\mathrm{t}, J=2.5 \mathrm{~Hz}, 1 \mathrm{H}), 3.80(\mathrm{~s}, 3 \mathrm{H}), 2.87-2.74(\mathrm{~m}, 2 \mathrm{H})$, 2.73-2.66 (m, 1H), $2.32(\mathrm{~s}, 3 \mathrm{H}), 2.29-2.22(\mathrm{~m}, 1 \mathrm{H}), 1.92-1.82(\mathrm{~m}$, $2 \mathrm{H}) ;{ }^{13} \mathrm{C}$ NMR (101 MHz, $\left.\mathrm{CDCl}_{3}\right) \delta$ 204.9, 199.4, 158.6, 139.9, 135.6, 132.4, 130.2, 129.9, 129.2, 128.3, 128.2, 113.7, 77.2, 55.2, 34.9, 31.7, 27.3, 24.9; IR $\left(\mathrm{CH}_{2} \mathrm{Cl}_{2}\right): 3059,2968,2837,1684,1605$, 1511, 1446, 1251, 1177, 1032, 880, 829, $701 \mathrm{~cm}^{-1}$; MS (EI): $\mathrm{m} / z$ $(\%)=334(25)\left[\mathrm{M}^{+}\right], 292(50), 291(34), 229(100), 187(33), 135(26)$, 121(31), 105(89), 77(57), 43(51); HRMS (EI) $\mathrm{m} / \mathrm{z}$ calcd for $\mathrm{C}_{22} \mathrm{H}_{22} \mathrm{O}_{3}$ 334.1569; found 334.1574.

4-(((1E)-2-Acetyl-2-benzoylcyclopentylidene)methyl)benzonitrile (45)

Prepared in reaction of 2-(pent-4-ynyl)-1-phenylbutane-1,3dione and 4-brombenzonitrile following modified general procedure (run at $80^{\circ} \mathrm{C}$ ) (51 mg, yield $40 \%$, isomer $\left.E / Z 70: 30\right)$. Product was isolated as oil after column chromatography on silica gel (25 g, hex/AcOEt $90: 10) .{ }^{1} \mathrm{H}$ NMR (400 MHz, $\mathrm{CDCl}_{3}$ ) $\delta$ 7.77-7.73 (m, 2H), 7.65-7.58 (m, 2H), 7.46-7.38 (m, 5H), 6.45 $(\mathrm{t}, J=2.6 \mathrm{~Hz}, 1 \mathrm{H}), 2.88-2.69(\mathrm{~m}, 4 \mathrm{H}), 2.31(\mathrm{~s}, 3 \mathrm{H}), 1.95-1.82(\mathrm{~m}$, $2 \mathrm{H}) ;{ }^{13} \mathrm{C}$ NMR $\left(101 \mathrm{MHz}, \mathrm{CDCl}_{3}\right) \delta$ 204.3, 198.2, 145.9, 141.9, $135.3,132.8,132.1,132.0,130.3,129.2,129.1,128.9,128.6$, 128.5, 127.1, 35.0, 32.1, 27.4, 24.8; indicative signals of minor isomer $(Z):{ }^{1} \mathrm{H}$ NMR $\left(400 \mathrm{MHz}, \mathrm{CDCl}_{3}\right) \delta 7.57(\mathrm{~d}, J=1.9 \mathrm{~Hz}, 1 \mathrm{H})$, 7.56-7.50 (m, 5H), 7.36-7.30 (m, 3H), 5.41 (p, $J=2.0 \mathrm{~Hz}, 1 \mathrm{H})$, 2.58-2.35 (m, 6H), $2.24(\mathrm{~s}, 3 \mathrm{H}) ;{ }^{13} \mathrm{C}$ NMR (101 MHz, $\mathrm{CDCl}_{3}$ ) $\delta$ 206.5, 199.4, 145.3, 135.2, 133.6, 133.0, 118.8, 110.3, 79.3, 35.4, 33.3, 31.1, 27.3; IR $\left(\mathrm{CH}_{2} \mathrm{Cl}_{2}\right): 3058,2962,2226,1695,1692,1603$, 1446, 1357, 1232, 700, $553 \mathrm{~cm}^{-1}$; MS (EI): $m / z(\%)=329(1)\left[\mathrm{M}^{+}\right]$, 287(39), 286(16), 258(8), 153(9), 127(6), 116(12), 105(100), 77(48), 51(15), 43(30); HRMS (EI): $m / z$ calcd for $\mathrm{C}_{22} \mathrm{H}_{19} \mathrm{NO}_{2}$ 329.1416; found 329.1404.

\section{((2E)-1-Benzoyl-2-benzylidenecyclopentyl)(phenyl)methanone}

(46)

Prepared in reaction of 2-(pent-4-ynyl)-1,3-diphenylpropane-1,3dione and bromobenzene following modified general procedure (run at $80{ }^{\circ} \mathrm{C}$ ) $(71 \mathrm{mg}$, yield $49 \%)$. Product was isolated as oil after column chromatography on silica gel $(25 \mathrm{~g}$, hex/AcOEt $95: 5) .{ }^{1} \mathrm{H}$ NMR $\left(400 \mathrm{MHz}, \mathrm{CDCl}_{3}\right) \delta 7.85-7.81(\mathrm{~m}, 4 \mathrm{H}), 7.50-$ $7.45(\mathrm{~m}, 2 \mathrm{H}), 7.41-7.36(\mathrm{~m}, 4 \mathrm{H}), 7.34-7.28(\mathrm{~m}, 4 \mathrm{H}), 7.24-7.20$ $(\mathrm{m}, 1 \mathrm{H}), 6.41(\mathrm{t}, J=2.5 \mathrm{~Hz}, 1 \mathrm{H}), 2.92(\mathrm{td}, J=7.4,2.5 \mathrm{~Hz}, 2 \mathrm{H})$, $2.68(\mathrm{t}, J=7.2 \mathrm{~Hz}, 2 \mathrm{H}), 1.89$ (p, $J=7.3 \mathrm{~Hz}, 2 \mathrm{H}) ;{ }^{13} \mathrm{C} \mathrm{NMR}(101$ $\left.\mathrm{MHz}, \mathrm{CDCl}_{3}\right) \delta 199.0,143.1,137.6,136.2,132.4,129.3,128.9$, 128.8, 128.4, 128.1, 126.8, 75.5, 36.7, 32.0, 24.2; IR $\left(\mathrm{CH}_{2} \mathrm{Cl}_{2}\right)$ : 3059, 3026, 2959, 1689, 1659, 1597, 1447, 1264, 1125, $697 \mathrm{~cm}^{-1}$; MS (EI): $m / z(\%)=366(2)\left[\mathrm{M}^{+}\right], 262(7), 261(20), 245(12), 244(21)$,
183(4), 155(6), 128(7), 115(7), 105(100), 91(13), 77(44), 51(11); HRMS (EI): $m / z$ calcd for $\mathrm{C}_{26} \mathrm{H}_{22} \mathrm{O}_{2} 366.1620$; found 366.1620 .

\section{((2E)-1-Benzoyl-2-(4-methoxybenzylidene)cyclopentyl)(phenyl)} methanone (47)

Prepared in reaction of 2-(pent-4-ynyl)-1,3-diphenylpropane-1,3dione and 4-bromoanisole following modified general procedure (run at $\left.80{ }^{\circ} \mathrm{C}\right)(88 \mathrm{mg}$, yield $56 \%)$. Product was isolated as oil after column chromatography on silica gel ( $25 \mathrm{~g}$, hex/AcOEt/ dioxane 85 : $10: 5) .{ }^{1} \mathrm{H}$ NMR (400 $\left.\mathrm{MHz}, \mathrm{CDCl}_{3}\right) \delta 7.84-7.80(\mathrm{~m}$, $4 \mathrm{H}), 7.49-7.43(\mathrm{~m}, 2 \mathrm{H}), 7.40-7.34(\mathrm{~m}, 4 \mathrm{H}), 7.27-7.22(\mathrm{~m}, 2 \mathrm{H})$, 6.89-6.84 (m, 2H), $6.34(\mathrm{t}, J=2.5 \mathrm{~Hz}, 1 \mathrm{H}), 3.78$ (s, 3H), 2.92-2.87 $(\mathrm{m}, 2 \mathrm{H}), 2.65(\mathrm{t}, J=7.2 \mathrm{~Hz}, 2 \mathrm{H}), 1.88(\mathrm{p}, J=7.3 \mathrm{~Hz}, 2 \mathrm{H}) ;{ }^{13} \mathrm{C} \mathrm{NMR}$ $\left(101 \mathrm{MHz}, \mathrm{CDCl}_{3}\right) \delta 199.2,158.4,140.8,136.2,132.3,130.3$, 130.0, 129.3, 128.3, 128.3, 113.5, 75.5, 55.1, 36.7, 31.9, 24.3; IR $\left(\mathrm{CH}_{2} \mathrm{Cl}_{2}\right): 3058,2956,2836,1687,1659,1606,1510,1251,1177$, 1033, 828, 701, $531 \mathrm{~cm}^{-1}$; MS (EI): $m / z(\%)=397(5)\left[\mathrm{M}^{+}\right], 369(15)$, 292(33), 291(100), 274(25), 263(19), 155(11), 135(19), 105(75), 91(13), 77(54), 51(15); HRMS (EI): $m / z$ calcd for $\mathrm{C}_{27} \mathrm{H}_{24} \mathrm{O}_{3}$ 396.1725; found 396.1719 .

\section{4-(((1E)-2,2-Dibenzoylcyclopentylidene)methyl)benzonitrile} (48)

Prepared in reaction of 2-(pent-4-ynyl)-1,3-diphenylpropane-1,3dione and 4-bromobenzonitrile following modified general procedure (run at $80{ }^{\circ} \mathrm{C}$ ) (63 mg, yield $40 \%$ ). Product was isolated as oil after column chromatography on silica gel ( $25 \mathrm{~g}$, hex/ AcOEt 90 : 10). ${ }^{1} \mathrm{H}$ NMR (400 MHz, $\left.\mathrm{CDCl}_{3}\right) \delta 7.81-7.76(\mathrm{~m}, 4 \mathrm{H})$, 7.59-7.56 (m, 2H), 7.50-7.45 (m, 2H), 7.40-7.34 (m, 6H), $6.38(\mathrm{t}, J$ $=2.6 \mathrm{~Hz}, 1 \mathrm{H}), 2.88(\mathrm{td}, J=7.4,2.6 \mathrm{~Hz}, 2 \mathrm{H}), 2.69(\mathrm{t}, J=7.2 \mathrm{~Hz}$, $2 \mathrm{H}), 1.90(\mathrm{p}, J=7.3 \mathrm{~Hz}, 2 \mathrm{H}) ;{ }^{13} \mathrm{C} \mathrm{NMR}\left(101 \mathrm{MHz}, \mathrm{CDCl}_{3}\right) \delta 198.5$, 147.3 , 142.0, 135.8, 132.6, 131.9, 129.3, 129.2, 128.5, 128.5, 127.2, 118.9, 110.1, 75.7, 36.6, 32.2, 24.2; IR $\left(\mathrm{CH}_{2} \mathrm{Cl}_{2}\right): 3361$, 3060, 2961, 2226, 1659, 1601, 1446, 1265, 1225, 1178, 879, 832, 736, 701, $554 \mathrm{~cm}^{-1}$; MS (EI): $m / z(\%)=391(1)\left[\mathrm{M}^{+}\right], 287(2)$, 285(7), 269(4), 201(6), 153(3), 130(4), 105(100), 77(40), 51(10); HRMS (EI): $m / z$ calcd for $\mathrm{C}_{27} \mathrm{H}_{21} \mathrm{NO}_{2} 391.1572$; found 391.1586 .

\section{Ethyl (2E)-2-benzylidene-1-(diethylphosphono) cyclopentanecarboxylate (49)}

Prepared in reaction of ethyl 2-(diethylphosphono)hept-6ynoate and bromobenzene following general procedure (20 mg, yield 14\%). Product was isolated as oil after HPLC chromatography (DCM/MeOH 99,5:0,5 $\rightarrow 99: 1) .{ }^{1} \mathrm{H}$ NMR $\left(400 \mathrm{MHz}, \mathrm{CDCl}_{3}\right) \delta 7.36-7.29(\mathrm{~m}, 4 \mathrm{H}), 7.27-7.17(\mathrm{~m}, 1 \mathrm{H}), 6.98$ (7.01-6.94 (m, 1H), 4.28-4.10 (m, 6H), 2.79-2.68 (m, 1H), 2.69$2.59(\mathrm{~m}, 1 \mathrm{H}), 2.58-2.45(\mathrm{~m}, 1 \mathrm{H}), 2.45-2.33(\mathrm{~m}, 1 \mathrm{H}), 2.01-1.87$ (m, 1H), 1.84-1.73 (m, 1H), 1.34-1.25 (m, 9H); ${ }^{13} \mathrm{C}$ NMR (101 $\left.\mathrm{MHz} \mathrm{CDCl}_{3}\right) \delta 170.5,140.3(\mathrm{~d}, J=7.7 \mathrm{~Hz}), 137.9(\mathrm{~d}, J=3.9 \mathrm{~Hz})$, $128.7(\mathrm{~d}, J=1.8 \mathrm{~Hz}), 128.2,127.5$ (d, $J=7.5 \mathrm{~Hz}), 126.7,63.3(\mathrm{~d}, J$ $=6.9 \mathrm{~Hz}), 62.9(\mathrm{~d}, J=7.3 \mathrm{~Hz}), 61.6,58.9(\mathrm{~d}, J=143.8 \mathrm{~Hz}), 33.7$ $(\mathrm{d}, J=3.4 \mathrm{~Hz}), 32.7(\mathrm{~d}, J=5.3 \mathrm{~Hz}), 29.7,25.4(\mathrm{~d}, J=6.3 \mathrm{~Hz}), 16.5$ $(\mathrm{d}, J=5.7 \mathrm{~Hz}), 14.0 ;{ }^{31} \mathrm{P}$ NMR $\left(162 \mathrm{MHz}, \mathrm{CDCl}_{3}\right) \delta 23.4$; IR $\left(\mathrm{CH}_{2} \mathrm{Cl}_{2}\right): 3233,2979,2928,1728,1446,1248,1025,966,759$, 698, $572 \mathrm{~cm}^{-1}$; MS (EI): $m / z(\%)=367(16), 366(41)\left[\mathrm{M}^{+}\right], 293(33)$, 229(42), 184(33), 183(82), 156(30), 155(100), 129(26), 115(29), 
105(23), 91(38), 77(25), 43(8); HRMS (EI): $m / z$ calcd for $\mathrm{C}_{19} \mathrm{H}_{27} \mathrm{O}_{5} \mathrm{P}$ 366.1596; found 366.1604 .

\section{Dipropan-2-yl ((2E)-2-benzylidene-1-cyanocyclopentyl) phosphonate (50)}

Prepared in reaction of dipropan-2-yl (1-cyanohex-5-yn-1-yl) phosphonate and bromobenzene following general procedure (88 mg, yield 63\%). Product was isolated as oil after column chromatography on silica gel (15 g, hex/AcOEt $60: 40) .{ }^{1} \mathrm{H}$ NMR $\left(400 \mathrm{MHz}, \mathrm{CDCl}_{3}\right) \delta$ 7.37-7.28 (m, 4H), 7.27-7.18 (m, 1H), 6.98$6.91(\mathrm{~m}, 1 \mathrm{H}), 4.86-4.73(\mathrm{~m}, 2 \mathrm{H}), 2.80-2.61$ (m, 2H), 2.54-2.41 $(\mathrm{m}, 1 \mathrm{H}), 2.37-2.25(\mathrm{~m}, 1 \mathrm{H}), 2.14-2.03(\mathrm{~m}, 1 \mathrm{H}), 1.89-1.77(\mathrm{~m}$, 1H), 1.39-1.27 (m, 12H); ${ }^{13} \mathrm{C} \mathrm{NMR}\left(101 \mathrm{MHz}, \mathrm{CDCl}_{3}\right) \delta 138.1$ (d, $=8.1 \mathrm{~Hz}), 136.5(\mathrm{~d}, J=3.7 \mathrm{~Hz}), 128.5(\mathrm{~d}, J=2.0 \mathrm{~Hz}), 128.3(\mathrm{~d}, J=$ $7.7 \mathrm{~Hz}), 128.2,127.2,119.8(\mathrm{~d}, J=6.4 \mathrm{~Hz}), 73.0(\mathrm{~d}, J=7.2 \mathrm{~Hz})$, $73.0(\mathrm{~d}, J=7.2 \mathrm{~Hz}), 46.1(\mathrm{~d}, J=148.1 \mathrm{~Hz}), 34.6(\mathrm{~d}, J=4.6 \mathrm{~Hz})$, $31.3(\mathrm{~d}, J=3.6 \mathrm{~Hz}), 24.8(\mathrm{~d}, J=4.1 \mathrm{~Hz}), 24.0(\mathrm{~d}, J=3.3 \mathrm{~Hz}), 24.0$ $(\mathrm{d}, J=3.3 \mathrm{~Hz}), 23.6(\mathrm{~d}, J=3.9 \mathrm{~Hz}), 23.5(\mathrm{~d}, J=3.8 \mathrm{~Hz}) ;{ }^{31} \mathrm{P}$ NMR $\left(162 \mathrm{MHz}, \mathrm{CDCl}_{3}\right) \delta$ 16.7; IR $\left(\mathrm{CH}_{2} \mathrm{Cl}_{2}\right): 3458,3253,2981,2936$, 2235, 1450, 1387, 1255, 1103, 989, 762, 696, $585 \mathrm{~cm}^{-1}$; MS (EI): $m / z(\%)=348(3), 347(10)\left[\mathrm{M}^{+}\right], 305(9), 264(20), 263(68), 210(10)$, 183(50), 182(100), 181(12), 166(32), 155(22), 129(19), 115(22), 91(25), 77(15), 51(7); HRMS (EI): $m / z$ calcd for $\mathrm{C}_{19} \mathrm{H}_{26} \mathrm{NO}_{3} \mathrm{P}$ 347.1650; found 347.1647 .

\section{Diethyl ((2E)-1-acetyl-2-benzylidenecyclopentyl)phosphonate} (51)

Prepared in reaction of diethyl (2-oxooct-7-yn-3-yl)phosphonate and bromobenzene following general procedure $(60 \mathrm{mg}$, yield $45 \%$ ). Product was isolated as oil after column chromatography on silica gel (15 g, hex/AcOEt/dioxane $45: 45: 10) .{ }^{1} \mathrm{H}$ NMR (400 $\left.\mathrm{MHz} \mathrm{CDCl}_{3}\right) \delta 7.36-7.31(\mathrm{~m}, 4 \mathrm{H}), 7.26-7.18(\mathrm{~m}, 1 \mathrm{H}), 7.01-6.96$ (m, 1H), 4.22-4.05 (m, 4H), 2.74-2.64 (m, 2H), 2.51-2.38 (m, $1 \mathrm{H}), 2.37(\mathrm{~s}, 3 \mathrm{H}), 2.35-2.23(\mathrm{~m}, 1 \mathrm{H}), 1.96-1.83(\mathrm{~m}, 1 \mathrm{H}), 1.77-1.65$ (m, 1H), 1.33-1.29 (m, 3H), $1.26(\mathrm{td}, J=7.1,0.6 \mathrm{~Hz}, 3 \mathrm{H}) ;{ }^{13} \mathrm{C}$ NMR $\left(101 \mathrm{MHz}, \mathrm{CDCl}_{3}\right) \delta 203.8,140.5(\mathrm{~d}, J=6.6 \mathrm{~Hz}), 137.6(\mathrm{~d}, J$ $=3.7 \mathrm{~Hz}), 128.6(\mathrm{~d}, J=1.8 \mathrm{~Hz}), 128.2,128.2(\mathrm{~d}, J=7.4 \mathrm{~Hz}), 126.8$, $66.7(\mathrm{~d}, J=139.8 \mathrm{~Hz}), 63.3(\mathrm{~d}, J=7.0 \mathrm{~Hz}), 62.5(\mathrm{~d}, J=7.3 \mathrm{~Hz})$, $32.7(\mathrm{~d}, J=0.9 \mathrm{~Hz}), 32.6(\mathrm{~d}, J=4.2 \mathrm{~Hz}), 27.5,25.0,24.9(\mathrm{~d}, J=6.9$ $\mathrm{Hz}), 16.4$ (d, $J=5.8 \mathrm{~Hz}), 16.3$ (d, $J=5.8 \mathrm{~Hz}) ;{ }^{31} \mathrm{P} \mathrm{NMR}(162 \mathrm{MHz}$, $\left.\mathrm{CDCl}_{3}\right) \delta 23.8,23.8 ;$ IR $\left(\mathrm{CH}_{2} \mathrm{Cl}_{2}\right): 3455,3230,2980,1706,1445$, 1227, 1049, 1024, 956, 760, 698, $599 \mathrm{~cm}^{-1}$; MS (EI): $\mathrm{m} / z(\%)=$ 336(4)[M+], 295(29), 294(100), 266(15), 237(13), 220(5), 156(31), 155(84), 128(22), 115(19), 105(11), 91(29), 77(16), 43(29); HRMS (EI): $m / z$ calcd for $\mathrm{C}_{18} \mathrm{H}_{25} \mathrm{O}_{4} \mathrm{P} 336.1490$; found 336.1502.

\section{(2E)-2-Benzylidene-1-(diphenylphosphoryl)cyclopentane-} carbonitrile (52)

Prepared in reaction of 2-(diphenylphosphoryl)hept-6-ynenitrile and bromobenzene following general procedure $(62 \mathrm{mg}$, yield $41 \%$ ). Product was isolated as oil after column chromatography on silica gel (15 g, hex/AcOEt $50: 50) .{ }^{1} \mathrm{H}$ NMR (400 MHz, $\mathrm{CDCl}_{3}$ ) б 8.28-8.18 (m, 2H), 8.05-7.96 (m, 2H), 7.67-7.52 (m, 4H), 7.51$7.41(\mathrm{~m}, 2 \mathrm{H}), 7.35-7.26(\mathrm{~m}, 2 \mathrm{H}), 7.26-7.18(\mathrm{~m}, 1 \mathrm{H}), 7.15(\mathrm{~d}, J=$ $7.2 \mathrm{~Hz}, 2 \mathrm{H}), 5.97-5.91(\mathrm{~m}, 1 \mathrm{H}), 2.89-2.70(\mathrm{~m}, 2 \mathrm{H}), 2.65-2.51(\mathrm{~m}$, $1 \mathrm{H}), 2.44-2.29(\mathrm{~m}, 1 \mathrm{H}), 2.23-2.11(\mathrm{~m}, 1 \mathrm{H}), 1.90-1.75(\mathrm{~m}, 1 \mathrm{H})$;
${ }^{13} \mathrm{C} \mathrm{NMR}\left(101 \mathrm{MHz}, \mathrm{CDCl}_{3}\right) \delta 138.6(\mathrm{~d}, J=6.6 \mathrm{~Hz}), 136.3(\mathrm{~d}, J=$ $3.4 \mathrm{~Hz}), 132.8(\mathrm{~d}, J=8.4 \mathrm{~Hz}), 132.7(\mathrm{~d}, J=2.9 \mathrm{~Hz}), 132.6(\mathrm{~d}, J=$ $2.6 \mathrm{~Hz}), 131.9$ (d, $J=8.5 \mathrm{~Hz}), 129.9(\mathrm{~d}, J=97.2 \mathrm{~Hz}), 128.85(\mathrm{~d}, J=$ $11.7 \mathrm{~Hz}), 128.78(\mathrm{~d}, J=6.6 \mathrm{~Hz}), 128.5(\mathrm{~d}, J=1.9 \mathrm{~Hz}), 128.4(\mathrm{~d}, J=$ $101.2 \mathrm{~Hz}), 128.21$ (d, $J=12.4 \mathrm{~Hz}), 128.15,127.3,121.6(\mathrm{~d}, J=2.8$ $\mathrm{Hz}), 47.5$ (d, $J=64.7 \mathrm{~Hz}), 34.4,31.8,25.3(\mathrm{~d}, J=2.2 \mathrm{~Hz}) ;{ }^{31} \mathrm{P} \mathrm{NMR}$ $\left(162 \mathrm{MHz}, \mathrm{CDCl}_{3}\right) \delta 30.4$; IR $\left(\mathrm{CH}_{2} \mathrm{Cl}_{2}\right): 3057,2961,2871,2230$, 1438, 1203, 1115, 725, 695, 607, 548, $526 \mathrm{~cm}^{-1}$; MS (EI): $\mathrm{m} / z(\%)$ $=384(12), 383(28)\left[\mathrm{M}^{+}\right], 382(6), 258(4), 202(25), 201(100)$, 182(15), 154(9), 115(7), 91(8), 77(25), 51(13); HRMS (EI): $\mathrm{m} / \mathrm{z}$ calcd for $\mathrm{C}_{25} \mathrm{H}_{22} \mathrm{NOP} 383.1439$; found 383.1428.

\section{Ethyl (2E)-2-benzylidene-1-(diphenylphosphoryl) cyclopentanecarboxylate (53)}

Prepared in reaction of ethyl 2-(diphenylphosphoryl)hept-6ynoate and bromobenzene following general procedure (83 mg, yield 52\%). Product was isolated as oil after column chromatography on silica gel $(15 \mathrm{~g}$, hex/AcOEt $60: 40 \rightarrow$ 50 : 50). ${ }^{1} \mathrm{H}$ NMR (400 MHz, $\left.\mathrm{CDCl}_{3}\right) \delta 7.97-7.89(\mathrm{~m}, 2 \mathrm{H}), 7.88-$ $7.80(\mathrm{~m}, 2 \mathrm{H}), 7.54-7.47$ (m, 2H), 7.46-7.38 (m, 4H), 7.34-7.28 $(\mathrm{m}, 2 \mathrm{H}), 7.24-7.18(\mathrm{~m}, 3 \mathrm{H}), 6.50(\mathrm{p}, J=7.0 \mathrm{~Hz}, 1 \mathrm{H}), 4.22-4.06$ (m, 2H), 2.69-2.51 (m, 2H), 2.47-2.34 (m, 1H), 1.85-1.68 (m, $2 \mathrm{H}), 1.28-1.22(\mathrm{~m}, 1 \mathrm{H}), 1.11(\mathrm{t}, J=7.1 \mathrm{~Hz}, 3 \mathrm{H}) ;{ }^{13} \mathrm{C}$ NMR (101 $\left.\mathrm{MHz} \mathrm{CDCl}_{3}\right) \delta 171.0(\mathrm{~d}, J=2.0 \mathrm{~Hz}), 140.5(\mathrm{~d}, J=6.5 \mathrm{~Hz}), 137.6$ $(\mathrm{d}, J=3.2 \mathrm{~Hz}), 133.1$ (d, $J=8.7 \mathrm{~Hz}), 132.2(\mathrm{~d}, J=8.7 \mathrm{~Hz}), 131.9$ $(\mathrm{d}, J=97.4 \mathrm{~Hz}), 131.68(\mathrm{~d}, J=2.8 \mathrm{~Hz}), 131.65(\mathrm{~d}, J=2.8 \mathrm{~Hz})$, 131.1 (d, $J=100.3 \mathrm{~Hz}), 128.63,128.61,128.56$ (d, $J=7.0 \mathrm{~Hz}$ ), $128.2(\mathrm{~d}, J=11.7 \mathrm{~Hz}), 128.1,127.7(\mathrm{~d}, J=11.7 \mathrm{~Hz}), 61.6,61.2(\mathrm{~d}, J$ $=65.9 \mathrm{~Hz}), 33.4,33.1(\mathrm{~d}, J=2.9 \mathrm{~Hz}), 25.7(\mathrm{~d}, J=5.1 \mathrm{~Hz}), 13.7 ;{ }^{31} \mathrm{P}$ NMR (162 MHz, $\left.\mathrm{CDCl}_{3}\right) \delta 34.1,31.2$; IR $\left(\mathrm{CH}_{2} \mathrm{Cl}_{2}\right): 3431,3057$, 2959, 1721, 1438, 1228, 1113, 724, 697, $549 \mathrm{~cm}^{-1}$; MS (EI): $\mathrm{m} / z$ $(\%)=431(18), 430(36)\left[\mathrm{M}^{+}\right], 357(10), 301(7), 288(6), 229(12)$, 219(32), 202(59), 201(100), 184(68), 183(46), 155(59), 129(24), 105(23), 91(32), 77(25), 43(8); HRMS (EI): $\mathrm{m} / \mathrm{z}$ calcd for $\mathrm{C}_{27} \mathrm{H}_{27} \mathrm{O}_{3} \mathrm{P}$ 430.1698; found 430.1705 .

\section{1-((2E)-2-Benzylidene-1-(diphenylphosphoryl)cyclopentyl) ethanone (54)}

Prepared in reaction of 3-(diphenylphosphoryl)oct-7-yn-2-one and bromobenzene following general procedure $(81 \mathrm{mg}$, yield $51 \%)$. Product was isolated as oil after column chromatography on silica gel (15 g, hex/AcOEt/dioxane $45: 45: 10) .{ }^{1} \mathrm{H}$ NMR (400 $\left.\mathrm{MHz} \mathrm{CDCl}_{3}\right) \delta$ 7.94-7.79 (m, 4H), 7.54-7.46 (m, 2H), 7.46-7.38 (m, 4H), 7.36-7.30 (m, 2H), 7.27-7.19 (m, 3H), 6.68 (s, 1H), 2.75$2.65(\mathrm{~m}, 1 \mathrm{H}), 2.62-2.52(\mathrm{~m}, 2 \mathrm{H}), 2.42(\mathrm{~s}, 3 \mathrm{H}), 2.38-2.28(\mathrm{~m}, 1 \mathrm{H})$, 1.76-1.63 (m, 2H); $\left.{ }^{13} \mathrm{C} \mathrm{NMR} \mathrm{(101} \mathrm{MHz,} \mathrm{CDCl}_{3}\right) \delta 205.3,140.8(\mathrm{~d}$, $J=5.7 \mathrm{~Hz}), 137.5(\mathrm{~d}, J=2.8 \mathrm{~Hz}), 133.0(\mathrm{~d}, J=9.2 \mathrm{~Hz}), 132.6(\mathrm{~d}, J$ $=8.6 \mathrm{~Hz}), 131.8(\mathrm{~d}, J=2.7 \mathrm{~Hz}), 131.7(\mathrm{~d}, J=2.7 \mathrm{~Hz}), 131.6(\mathrm{~d}, J=$ $97.8 \mathrm{~Hz}$ ), 131.0 (d, $J=97.8 \mathrm{~Hz}), 129.3(\mathrm{~d}, J=6.5 \mathrm{~Hz}), 128.6$, $128.21,128.17$ (d, $J=11.4 \mathrm{~Hz}), 128.0$ (d, $J=11.9 \mathrm{~Hz}), 127.0,68.8$ $(\mathrm{d}, J=64.4 \mathrm{~Hz}), 32.8(\mathrm{~d}, J=3.7 \mathrm{~Hz}), 32.7,28.3,25.4(\mathrm{~d}, J=5.5$ $\mathrm{Hz}) ;{ }^{31} \mathrm{P}$ NMR $\left(162 \mathrm{MHz}, \mathrm{CDCl}_{3}\right) \delta 35.80,34.60 ;$ IR $\left(\mathrm{CH}_{2} \mathrm{Cl}_{2}\right)$ : 3378, 3058, 2960, 2925, 2854, 1699, 1437, 1179, 1112, 750, 722, 696, $543 \mathrm{~cm}^{-1}$; MS (EI): $m / z(\%)=400(24)\left[\mathrm{M}^{+}\right], 359(42)$, 358(100), 281(16), 219(24), 202(47), 201(98), 182(45), 167(53), 
155(40), 128(34), 115(25), 105(21), 91(36), 77(63), 51(33), 43(65); HRMS (EI): $m / z$ calcd for $\mathrm{C}_{26} \mathrm{H}_{25} \mathrm{O}_{2} \mathrm{P} 400.1592$; found 400.1587 .

\section{Dimethyl (2E)-2-(4-fluorobenzylidene)cyclopentane-1,1- dicarboxylate (55)}

Prepared in reaction of dimethyl 4-pentenylmalonate and 1chloro-4-fluorobenzene following modified general procedure (run at $80{ }^{\circ} \mathrm{C}$ ) $(74 \mathrm{mg}$, yield 63\%). Product was isolated as oil after column chromatography on silica gel $(15 \mathrm{~g}$, hex/AcOEt 90 : 10). ${ }^{1} \mathrm{H}$ NMR (400 MHz, $\left.\mathrm{CDCl}_{3}\right) \delta 7.33-7.27(\mathrm{~m}, 2 \mathrm{H}), 7.04-$ $6.97(\mathrm{~m}, 2 \mathrm{H}), 6.66(\mathrm{t}, J=2.7 \mathrm{~Hz}, 1 \mathrm{H}), 3.76(\mathrm{~s}, 6 \mathrm{H}), 2.66(\mathrm{td}, J=$ $7.2,2.6 \mathrm{~Hz}, 2 \mathrm{H}), 2.38(\mathrm{t}, J=6.9 \mathrm{~Hz}, 2 \mathrm{H}), 1.83(\mathrm{p}, J=7.1 \mathrm{~Hz}, 2 \mathrm{H})$; ${ }^{13} \mathrm{C}$ NMR $\left(101 \mathrm{MHz}, \mathrm{CDCl}_{3}\right) \delta 171.3,162.8,161.5(\mathrm{~d}, J=246.8$ $\mathrm{Hz}), 140.6$ (d, $J=2.1 \mathrm{~Hz}), 133.7(\mathrm{~d}, J=3.3 \mathrm{~Hz}), 130.2(\mathrm{~d}, J=8.0$ $\mathrm{Hz}$ ), 126.3, 115.0 (d, $J=21.5 \mathrm{~Hz}), 65.3,52.7,35.7,31.8,24.7 ;{ }^{19} \mathrm{~F}$ NMR (376 MHz, $\left.\mathrm{CDCl}_{3}\right) \delta-114.96 ;$ IR $\left(\mathrm{CH}_{2} \mathrm{Cl}_{2}\right)$ : 2954, 2879, 2842, 1733, 1603, 1508, 1434, 1227, 1190, 1159, 1098, 1065, 1014, 929, 885, 827, 773, $523 \mathrm{~cm}^{-1}$. MS (EI): $\mathrm{m} / z(\%)=293(6)$ $\left[\mathrm{M}^{+}\right], 292(32), 260(13), 233(32), 232(44), 201(65), 200(21)$, 173(100), 146(23), 133(18), 109(44), 77(7), 59(16), 43(4); HRMS (EI): $m / z$ calcd for $\mathrm{C}_{16} \mathrm{H}_{17} \mathrm{O}_{4} \mathrm{~F}$ 292.1111; found 292.1117.

\section{Dimethyl (2E)-2-(4-(methoxycarbonyl)benzylidene) cyclopentane-1,1-dicarboxylate (56)}

Prepared in reaction of dimethyl 4-pentenylmalonate and methyl 4-chlorobenzoate following modified general procedure (run at $80{ }^{\circ} \mathrm{C}$ ) (78 mg, yield 59\%, isomer $\left.E / Z 80: 20\right)$. Product was isolated as oil after column chromatography on silica gel $(25 \mathrm{~g}$, hex/AcOEt $90: 10 \rightarrow 85: 15) .{ }^{1} \mathrm{H}$ NMR (400 MHz, $\mathrm{CDCl}_{3}$ ) $\delta$ 7.99-7.95 (m, 2H), 7.40-7.36 (m, 2H), $6.72(\mathrm{t}, J=2.6 \mathrm{~Hz}, 1 \mathrm{H})$, $3.88(\mathrm{~s}, 3 \mathrm{H}), 3.76(\mathrm{~s}, 6 \mathrm{H}), 2.70(\mathrm{td}, J=7.2,2.6 \mathrm{~Hz}, 2 \mathrm{H}), 2.38(\mathrm{t}, J=$ $7.0 \mathrm{~Hz}, 2 \mathrm{H}), 1.83(\mathrm{p}, J=7.1 \mathrm{~Hz}, 2 \mathrm{H}) ;{ }^{13} \mathrm{C} \mathrm{NMR}\left(101 \mathrm{MHz}, \mathrm{CDCl}_{3}\right)$ $\delta 171.0,166.8,143.7,142.0,129.4,128.5,128.3,126.7,65.5,52.8$, $51.9,35.6,32.1,24.7$; indicative signals of minor isomer $(Z):{ }^{1} \mathrm{H}$ NMR $\left(400 \mathrm{MHz}, \mathrm{CDCl}_{3}\right) \delta 7.95-7.89(\mathrm{~m}, 2 \mathrm{H}), 7.35(\mathrm{~s}, 1 \mathrm{H}), 6.65(\mathrm{~d}$, $J=2.3 \mathrm{~Hz}, 1 \mathrm{H}), 3.87(\mathrm{~s}, 3 \mathrm{H}), 3.43(\mathrm{~s}, 5 \mathrm{H}), 2.67-2.62(\mathrm{~m}, 2 \mathrm{H}), 2.42$ $(\mathrm{t}, J=7.0 \mathrm{~Hz}, 2 \mathrm{H}), 1.78-1.70(\mathrm{~m}, 2 \mathrm{H}) ;{ }^{13} \mathrm{C} \mathrm{NMR}\left(101 \mathrm{MHz}, \mathrm{CDCl}_{3}\right)$ $\delta 170.7,143.5,141.4,129.0,128.3,128.2,125.8,63.8,52.4,39.4$, 34.8, 22.5, 14.0; IR $\left(\mathrm{CH}_{2} \mathrm{Cl}_{2}\right): 2963,2843,1724,1606,1565,1435$, 1279, 1183, 1156, 1111, 1066, 1017, 966, 890, 777, 700, $522 \mathrm{~cm}^{-1}$; MS (EI): $m / z(\%)=333(9)\left[\mathrm{M}^{+}\right], 332(39), 301(24), 273(24), 272(43)$, 242(32), 241(100), 240(48), 214(30), 213(98), 181(32), 155(35), 154(39), 153(48), 129(44), 128(19), 115(16), 105(6), 91(9), 77(12), 59(41), 41(4); HRMS (EI): $\mathrm{m} / z$ calcd for $\mathrm{C}_{18} \mathrm{H}_{20} \mathrm{O}_{6}$ 332.1260; found 332.1266 .

\section{Conclusions}

In summary, we developed an efficient protocol for tandem Pdcatalyzed intramolecular addition of active methylene compounds to alkynes, followed by subsequent cross-coupling with (hetero)aryl bromides and chlorides. The methodology features exceptional tolerance to functional groups (including unprotected $\mathrm{OH}, \mathrm{NH}_{2}$, or enolizable ketones), broad applicability of aryl and heteroaryl bromides of different electronic properties, as well as a range of active methylene partners, including acetylenic derivatives of malonates, cyanomalonates, $\beta$-ketoesters, $\beta$-diketones, cyanoacetates, and organophosphorus compounds. Mechanistic studies revealed a plausible mechanism comprising oxidative addition of haloarene, nucleophilic addition to alkyne activated by coordination to aryl-Pd(II), and reductive elimination. However, for the transformations of less $\mathrm{C}-\mathrm{H}$ acidic substrates (e.g. $\beta$-ketoesters, $\beta$ diketones) and electron-deficient haloarenes, an alternative path involving syn-carbometallation may operate in parallel.

\section{Conflicts of interest}

There are no conflicts to declare.

\section{Acknowledgements}

Financial support from the Polish National Science Centre (Grant decision DEC-2016/22/E/ST5/00537) is gratefully acknowledged.

\section{Notes and references}

1 X.-F. Wu, P. Anbarasan, H. Neumann and M. Beller, Angew. Chem., Int. Ed., 2010, 49, 9047-9050.

2 (a) D. E. Fogg and E. N. dos Santos, Coord. Chem. Rev., 2004, 248, 2365-2379; (b) R. P. Herrera and E. Marqués-López, Multicomponent Reactions: Concepts and Applications for Design and Synthesis, Wiley, 1st edn, 2015.

3 (a) S. Cacchi, J. Organomet. Chem., 1999, 576, 42-64; (b) G. Balme, E. Bossharth and N. Monteiro, Eur. J. Org. Chem., 2003, 2003, 4101-4111; (c) G. Balme, D. Bouyssi, T. Lomberget and N. Monteiro, Synthesis, 2003, 2115-2134; (d) F. Alonso, I. P. Beletskaya and M. Yus, Chem. Rev., 2004, 104, 3079-3160; (e) G. Balme, D. Bouyssi and N. Monteiro, Pure Appl. Chem., 2006, 78, 231-239; (f) G. Zeni and R. C. Larock, Chem. Rev., 2006, 106, 4644-4680; (g) F. Dénès, A. Pérez-Luna and F. Chemla, Chem. Rev., 2010, 110, 2366-2447; (h) H. Ohno, Asian J. Org. Chem., 2013, 2, 18-28; (i) R. Chinchilla and C. Nájera, Chem. Rev., 2014, 114, 1783-1826.

4 (a) G. Fournet, G. Balme and J. Gore, Tetrahedron Lett., 1987, 28, 4533-4536; (b) G. Fournet, G. Balme and J. Gore, Tetrahedron Lett., 1989, 30, 69-70.

5 (a) G. Fournet, G. Balme, B. Van Hemelryck and J. Gore, Tetrahedron Lett., 1990, 31, 5147-5150; (b) G. Fournet, G. Balme and J. Gore, Tetrahedron, 1991, 47, 6293-6304.

6 D. Bouyssi, G. Balme and J. Gore, Tetrahedron Lett., 1991, 32, 6541-6544.

7 G. Liu and X. Lu, Tetrahedron Lett., 2002, 43, 6791-6794.

8 W. Chaładaj and S. Domański, Adv. Synth. Catal., 2016, 358, 1820-1825.

9 D. Fujino, H. Yorimitsu and A. Osuka, Org. Lett., 2012, 14, 2914-2917.

10 (a) X.-H. Duan, L. Guo, H.-P. Bi, X.-Y. Liu and Y.-M. Liang, Org. Lett., 2006, 8, 3053-3056; (b) L.-N. Guo, X.-H. Duan, H.-P. Bi, X.-Y. Liu and Y.-M. Liang, J. Org. Chem., 2006, 71, 3325-3327; (c) D. Zhang, Z. Liu, E. K. Yum and 
R. C. Larock, J. Org. Chem., 2007, 72, 251-262; (d) Z.-H. Guan, Z.-H. Ren, L.-B. Zhao and Y.-M. Liang, Org. Biomol. Chem., 2008, 6, 1040-1045.

11 D. Fujino, H. Yorimitsu and K. Oshima, J. Am. Chem. Soc., 2011, 133, 9682-9685.

12 (a) A. Arcadi, S. Cacchi, R. C. Larock and F. Marinelli, Tetrahedron Lett., 1993, 34, 2813-2816; (b) A. Arcadi and E. Rossi, Tetrahedron Lett., 1996, 37, 6811-6814; (c) A. Arcadi, S. Cacchi, G. Fabrizi, F. Marinelli and L. M. Parisi, Tetrahedron, 2003, 59, 4661-4671; (d)
A. Kołodziejczyk and W. Chaładaj, Eur. J. Org. Chem., 2018, 2018, 2554-2560.

13 A. Kołodziejczyk, S. Domański and W. Chaładaj, J. Org. Chem., 2018, 83, 12887-12896.

14 See the ESI $\dagger$ for more details.

15 N. C. Bruno, M. T. Tudge and S. L. Buchwald, Chem. Sci., 2013, 4, 916-920.

16 (a) J. M. Fox, X. Huang, A. Chieffi and S. L. Buchwald, J. Am. Chem. Soc., 2000, 122, 1360-1370; (b) H. Tanaka, K. Isobe, S. Kawaguchi and S. Okeya, Bull. Chem. Soc. Jpn., 1984, 57, 1850-1855. 